\title{
LP Rounding for $k$-Centers with Non-uniform Hard Capacities
}

\author{
(Extended Abstract)
}

\author{
Marek Cygan* $\quad$ MohammadTaghi Hajiaghayi ${ }^{\dagger} \quad$ Samir Khuller $^{\ddagger}$
}

\begin{abstract}
In this paper we consider a generalization of the classical $k$-center problem with capacities. Our goal is to select $k$ centers in a graph, and assign each node to a nearby center, so that we respect the capacity constraints on centers. The objective is to minimize the maximum distance a node has to travel to get to its assigned center. This problem is $N P$-hard, even when centers have no capacity restrictions and optimal factor 2 approximation algorithms are known. With capacities, when all centers have identical capacities, a 6 approximation is known with no better lower bounds than for the infinite capacity version.

While many generalizations and variations of this problem have been studied extensively, no progress was made on the capacitated version for a general capacity function. We develop the first constant factor approximation algorithm for this problem. Our algorithm uses an LP rounding approach to solve this problem, and works for the case of non-uniform hard capacities, when multiple copies of a node may not be chosen and can be extended to the case when there is a hard bound on the number of copies of a node that may be selected. In addition we establish a lower bound on the integrality gap of 7(5) for nonuniform (uniform) hard capacities. In addition we prove that if there is a $(3-\epsilon)$-factor approximation for this problem then $P=N P$.

Finally, for non-uniform soft capacities we present a much simpler 11-approximation algorithm, which we find as one more evidence that hard capacities are much harder to deal with.
\end{abstract}

\footnotetext{
*IDSIA, University of Lugano, Switzerland, marek@idsia.ch. Research supported in part by ERC Starting Grant PAAl 259515 and Foundation for Polish Science.

${ }^{\dagger}$ University of Maryland, College Park MD 20742, ha jiagha@cs . umd. edu. Research supported in part by NSF CAREER award 1053605, ONR YIP award N000141110662, DARPA/AFRL award FA8650-11-1-7162, and a University of Maryland Research and Scholarship Award (RASA).

${ }^{\ddagger}$ Dept. of Computer Science, University of Maryland, College Park MD 20742, samir@Cs . umd. edu. Research supported by NSF CCF-0728839, NSF CCF-0937865 and a Google Research Award.
} 


\section{Introduction}

The $k$-center problem is a classical facility location problem and is defined as follows: given an edgeweighted graph $G=(V, E)$ find a subset $S \subseteq V$ of size at most $k$ such that each vertex in $V$ is "close" to some vertex in $S$. More formally, once we choose $S$ the objective function is $\max _{u \in V} \min _{v \in S} d(u, v)$, where $d$ is the distance function (a metric). The problem is known to be NP-hard [13]. Approximation algorithms for the $k$-center problem have been well studied and are known to be optimal [14, 17, 18, 19]. In this paper we consider the $k$-center problem with non-uniform capacities. We have a capacity function $L$ defined for each vertex, hence $L(u)$ denotes the capacity of vertex $u$. The goal is to identify a set $S$ of at most $k$ centers, as well as an assignment of vertices to "nearby" centers. No more than $L(u)$ vertices may be assigned to a chosen center at vertex $u$. Under these constraints we wish to minimize the maximum distance between a vertex $v$ and its assigned center $\phi(v)$. Formally, the cost of a solution $S$ is $\min _{S \subseteq V,|S|=k} \max _{v \in V} d(v, \phi(v))$ such that $|\{v \mid \phi(v)=u\}| \leq L(u) \forall u \in S$ where $\phi: V \rightarrow S$.

For the special case when all the capacities are identical, a 6 approximation was developed by Khuller and Sussmann [22] improving the previous bound of 10 by Bar-Ilan, Kortsarz and Peleg [2]. In the special case when multiple copies of the same vertex may be chosen, the approximation factor was improved to 5. No improvements have been obtained on these results in the last 15 years. The assumption that the capacities are identical is crucial for both these approaches as it allows one to select centers and then "shift" to a neighboring vertex. In addition, one can use arguments such as $\left\lceil\frac{N}{L}\right\rceil$ is a lower bound on the optimal solution; with non-uniform capacities we cannot use such a bound. This problem has resisted any progress at all, and no constant approximation algorithm was developed for the non-uniform capacity version.

In this work we present the first constant factor approximations for the $k$-center problem with arbitrary capacities. Moreover, our algorithm satisfies hard capacity constraints and only one copy of any vertex is chosen. When multiple copies of a vertex can be chosen then a constant factor approximation is implied by our result for the hard capacity version. For convenience, we discuss the algorithm for the case when at most one copy of a vertex may be chosen. Our algorithms use a novel LP rounding method to obtain the result. In fact this is the first time that LP techniques have been applied for any variation of the $k$-center problem.

While our constants are large, we do show via integrality gap examples that the problem with nonuniform capacities is significantly harder than the basic $k$-center problem. In addition we establish that if there is a $(3-\epsilon)$-approximation for the $k$-center problem with non-uniform capacity constraints then $P=N P$. Such a result is known for the cost $k$-center problem [8] and from that one can infer the result for the unit cost capacitated $k$-center problem with non-uniform capacities, but our reduction is a direct reduction from Exact Cover by 3 -Sets and considerably simpler. We would like to note that for the $k$ supplier problem, which can be seen as $k$-center with disjoint sets of clients and potential centers, a simple proof of $(3-\epsilon)$ approximation hardness under $P \neq N P$ was obtained by Karloff and can be found in [18].

In all cases of studying covering problems, the hard capacity restriction makes the problems very challenging. For example, for the simple capacitated vertex cover problem with soft capacities, a 2 approximation can be obtained by a variety of methods [15, 12] - however imposing a hard capacity restriction makes the problem as hard as set cover [9]. In the special case of unweighted graphs it was shown that a 3 approximation is possible [9], which was subsequently improved to 2 [11].

\subsection{Related Facility Location Work}

The facility location problem is a central problem in operations research and computer science and has been a testbed for many new algorithmic ideas resulting a number of different approximation algorithms. In this problem, given a metric (via a weighted graph $G$ ), a set of nodes called clients, and opening costs on some nodes called facilities, the goal is to open a subset of facilities such that the sum of their opening costs and connection costs of clients to their nearest open facilities is minimized. When the facilities have capacities, the problem is called the capacitated facility location problem. The first constant-factor 
approximation algorithm for the (uncapacitated) version of this problem was given by Shmoys, Tardos, and Aardal [30] and was based on LP rounding and a filtering technique due to Lin and Vitter [26]. A long series of improvements culminated in a 1.5 approximation due to Byrka [5]. Up to now, the best known approximation ratio is 1.488, due to $\mathrm{Li}$ [25] who uses a randomized selection in Byrka's algorithm [5]. Guha and Khuller [16] showed that this problem is hard to approximate within a factor better than 1.463 , assuming $N P \nsubseteq D T I M E\left[n^{O(\log \log n)}\right]$.

Capacitated facility location has also received a great deal of attention in recent years. Two main variants of the problem are soft-capacitated facility location and hard-capacitated facility location: in the latter problem, each facility is either opened at some location or not, whereas in the former, one may specify any integer number of facilities to be opened at that location. Soft capacities make the problem easier and by modifying approximation algorithms for the uncapacitated problems, we can also handle this case [30, 20]. Korupolu, Plaxton, and Rajaraman [23] gave the first constant-factor approximation algorithm that handles hard capacities, based on a local search procedure, but their approach works only if all capacities are equal. Chudak and Williamson [7] improved this performance guarantee to 5.83 for the same uniform capacity case. Pál, Tardos, and Wexler [28] gave the first constant performance guarantee for the case of non-uniform hard capacities. This was recently improved by Mahdian and Pál [27] and Zhang, Chen, and Ye [31] to yield a 5.83-approximation algorithm. All these approaches are based on local search. The only LP-relaxation based approach for this problem is due to Levi, Shmoys and Swamy [24] who gave a 5-approximation algorithm for the special case in which all facility opening costs are equal (otherwise the LP does not have a constant integrality gap). The above approximation algorithms for hard capacities are focused on the uniform demand case or the splittable case in which each unit of demand can be served by a different facility. Recently, Bateni and Hajiaghayi [4] considered the unsplittable hard-capacitated facility location problem when we allow violating facility capacities by a $1+\epsilon$ factor (otherwise, it is NP-hard to obtain any approximation factor) and obtain an $O(\log n)$ approximation algorithm for this problem.

A problem very close to both facility location and $k$-center is the $k$-median problem in which we want to open at most $k$ facilities (like in the $k$-center problem) and the goal is to minimize the sum of connection costs of clients to their nearest open facilities (like facility location). If facilities have capacities the problem is called capacitated $k$-median. The approaches for uncapacitated facility location often work for $k$-median. In particular, Charikar, Guha, Tardos, and Shmoys [6] gave the first constant factor approximation for $k$ median based on LP rounding. The best approximation factor for $k$-median is $3+\epsilon$, for an arbitrary positive constant $\epsilon$, via the local search algorithm of Arya et al. [1]. Unfortunately obtaining a constant factor approximation algorithm for capacitated $k$-median still remains open despite consistent effort. The methods used to solve uncapacitated $k$-median or even the local search technique for capacitated facility location all seem to suffer from serious drawbacks when trying to apply them for capacitated $k$-median. For example standard LP relaxation is known to have an unbounded integrality gap [6]. The only previous attempts with constant approximation factors for this problem violate the capacities within a constant factor for the uniform capacity case [6] and the non-uniform capacity case [10] or exceed the number $k$ of facilities by a constant factor [3].

Removing the metric: We employ the standard "thresholding" method used for bottleneck optimization problems. We can assume that we guess the optimal solution, since there are polynomially many distinct distances between pairs of nodes. Once we guess the distance correctly, we create an unweighted graph consisting of those edges $u v$ such that $d(u, v) \leq O P T$. We henceforth assume that we are considering the problem for an undirected graph $G$.

CAPACitated $k$-Center PRoblem

Input: An undirected graph $G=(V, E)$, a capacity function $L: V \rightarrow \mathbb{N}$ and an integer $k$.

Output: A set $S \subseteq V$ of size $k$, and a function $\phi: V \rightarrow S$, such that for each $u \in S,\left|\phi^{-1}(u)\right| \leq L(u)$. Goal: Minimize $\max _{v \in V} \operatorname{dist}_{G}(v, \phi(v))$. 
By a $c$-approximation algorithm we denote a polynomial time algorithm, that for an instance for which there exists a solution with objective function equal to 1 , returns a solution using distances at most $c$. Note that the distance function $\operatorname{dist}(u, v)$, measures the distance in the unweighted undirected graph.

In the soft-capacitated version $S$ can be a multiset, that is one can open more than one center at a vertex. To avoid confusion we call the standard version of the problem hard-capacitated.

\subsection{Our results}

While LP based algorithms have been widely used for uncapacitated facility location problems as well as capacitated versions of facility location with soft capacities, these methods are not of much use for problems in dealing with hard capacities due to the fact that they usually have an unbounded integrality gap [6, 28].

For general undirected graphs this is also the case for the capacitated $k$-center problem. Consider the LP relaxation for the natural IP, which we denote as LP1. We use $y_{u}$ as an indicator variable for open centers.

$$
\begin{aligned}
& \sum_{u \in V} y_{u}=k \\
& x_{u, v} \leq y_{u} \\
& \sum_{v \in V} x_{u, v} \leq L(u) y_{u} \\
& \sum_{u \in V} x_{u, v}=1 \\
& 0 \leq y_{u} \leq 1 \\
& x_{u, v}=0 \\
& x_{u, v} \geq 0
\end{aligned}
$$

$$
\begin{aligned}
\forall u, v & \in V \\
\forall u & \in V \\
\forall v & \in V \\
\forall u & \in V \\
\forall u, v \in V \operatorname{dist}_{G}(u, v) & >1 \\
\forall u, v & \in V
\end{aligned}
$$

For the sake of presentation we have introduced variables $x_{u, v}$ for all $u$, $v$, even if the distance between $u$ and $v$ in $G$ is greater than one. We will use those variables in our rounding algorithm. Furthermore in constraints (1) and (4) we used equality instead of inequality to make our rounding algorithm and lemma formulations simpler. In the soft-capacitated version the $y_{u} \leq 1$ part of constraint (5) should be removed. Note that we are only interested in feasilibity of LP1, and there is no objective function.

For an undirected graph $G=(V, E)$ and a positive integer $\delta$, by $G^{\delta}$ we denote the graph $\left(V, E^{\prime}\right)$, where $u v \in E^{\prime}$ iff $\operatorname{dist}_{G}(u, v) \leq \delta$. By an integrality gap of LP1 we mean the minimum positive integer $\delta$ such that if LP1 has a feasible solution, then the graph $G^{\delta}$ admits a capacitated $k$-center solution. As this is usually the case for capacitated problems, by a simple example we prove LP1 has unbounded integrality gap for general graphs.

Theorem 1. LP1 has unbounded integrality gap, even for uniform capacities.

Proof. Let $G^{\prime}$ be a graph that consists of two adjacent vertices $a, b$ together with 4 vertices adjacent to both of them (see Fig. 11). Set uniform capacity $L=4, k=3$ and consider the graph $G$ which is a disjoint union of two copies of $G^{\prime}$. Observe that by setting $y_{a}=y_{b}=0.75$ in each of the copies as well as $x_{a, v}=x_{b, v}=0.5$, for all six vertices $v$, we obtain a feasible solution to the LP relaxation. No matter what $\delta$ we choose, there is no capacitated $k$-center with $L=4$ in the graph $G^{\delta}$.

However, interestingly, if we assume that the given graph is connected, the situation changes dramatically. Our main result is, that both for hard and soft capacitated version of the $k$-center problem, even for non-uniform capacities, LP1 has constant integrality gap for connected graphs. Moreover by using novel techniques we show a corresponding polynomial time rounding algorithm, which consists of several steps, described at high level in the following subsection. The actual algorithm is somewhat complex, although it can be implemented quite efficiently. 


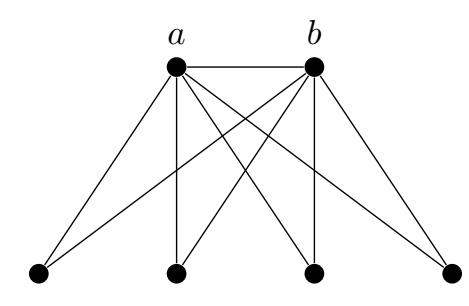

Figure 1: A single connected component of the graph $G$ used in the proof of Theorem 1 .

Theorem 2. There is a polynomial time algorithm, which given an instance of the hard-capacitated $k$ center problem for a connected graph, and a fractional feasible solution for LP1, can round it to an integral solution that uses non-zero $x_{u, v}$ variables for pairs of nodes with distance at most $c$.

Corollary 3. The integrality gap of LP1 for connected graphs is bounded by a constant, and there is a constant factor approximation algorithm for connected graphs.

To simplify the presentation we do not calculate the exact constant proved in the above corollary, but it is in the order of hundreds. As a counterposition, for soft capacities in Section 3 we present a much simpler 11-approximation algorithm, which we find as one more evidence that hard capacities are much harder to deal with.

Theorem 4. For connected graphs there is a polynomial time rounding algorithm, upper bounding the integrality gap of LP1 by 11 for soft-capacities.

By using standard techniques one can restrict the capacitated $k$-center problem to connected graphs.

Theorem 5. If there exists a polynomial time c-approximation algorithm for the (soft) capacitated k-center problem in connected graphs, then there exists a polynomial time c-approximation algorithm for general graphs.

Proof. Let us assume that we are given a graph $G$ with connected components $C_{1}, \ldots, C_{t}$, a capacity function $L: V \rightarrow \mathbb{N}$ and an integer $k$. For each connected component $C_{i}$ using binary search we find the smallest value of $k_{i} \leq k$ for which our black box algorithm finds a solution (if there is no solution for $k_{i}=k$ then we set $k_{i}=\infty$ ). If $\sum_{i=1}^{t} k_{i}>k$ then we answer NO, otherwise we return the union of solutions found by the black box algorithm.

To prove correctness of the above algorithm it is enough to observe that if there exists a solution $S$ to the $k$-center problem in the graph $G$ then for each connected component $C_{i}$ we have $k_{i} \leq\left|S \cap V\left(C_{i}\right)\right|$.

Therefore we prove there is a constant factor approximation algorithm for the hard-capacitated $k$-center problem 1 . Our results easily extend to the case when there is an upper bound $U(u)$ of the number of times vertex $u$ may be chosen as a center. Constraint 5 should be modified to be $0 \leq y_{u} \leq U(u)$ to yield a relaxation LP2. We can employ the same rounding procedure as discussed for the hard capacity case with $U(u)=1$.

The proof of the following theorem is omitted.

Theorem 6. There is a polynomial time algorithm, which given an instance of the hard-capacitated $k$ center problem for a connected graph, and a fractional feasible solution for LP2, can round it to an integral solution that uses non-zero $x_{u, v}$ variables for pairs of nodes with distance at most $c$.

\footnotetext{
${ }^{1}$ With some care, perhaps some of the constants can be improved, however our focus was to show that a constant approximation is obtainable using LP rounding.
} 
While our constants are large, we do show in Section 5 via integrality gap examples that the problem with non-uniform capacities is significantly harder than the basic $k$-center problem.

Theorem 7. For connected graphs the integrality gap of LP1 is at least 5 for uniform-hard-capacities and at least 4 for uniform-soft-capacities.

Moreover in the non-uniform hard-capacitated case, the integrality gap of LP1 for connected graphs is at least 7 , even if all the non-zero capacities are equal.

Despite the fact, that the algorithm of [22] for uniform capacities was obtained more than a decade ago, no lower bound for the capacity version (neither soft nor hard), better than the trivial $2-\epsilon$, derived from the uncapacitated version, is known. We believe that the integrality gap examples, presented in this paper, are of independent interest since they may help in proving a stronger lower bound for the capacitated $k$-center problem with uniform capacities.

To make a step in this direction we investigate lower bounds for the non-uniform case. By a reduction from the cost $k$-center problem [8] one can show that there is no $(3-\epsilon)$-approximation for the capacitated $k$-center problem with non-uniform capacities. By a simple reduction from Exact Cover by 3 -Sets, in Section 6, we prove the same result under the assumption $P \neq N P$.

Finally we give evidence that our LP approach might be the proper tool for solving the capacitated $k$-center problem. The proof of the following theorem shows that when the Khuller-Sussmann algorithm fails to find a solution then in fact there is no feasible LP solution for that guess of distance. The smallest radius guess for which the algorithm succeeds, proves an integrality gap on the LP. Considering the result of Theorem 7, as we show in Section 4, it follows that for uniform capacities the gap in the analysis is small, since our bounds are tight up to an additive +1 error.

Theorem 8. For connected graphs the integrality gap of LP1 is at most 6 for uniform-hard-capacities and at most 5 for uniform-soft-capacities.

\subsection{Our techniques}

We assume that $G$ is connected and that LP1 has a feasible solution for the graph $G$. We call two functions $x: V \times V \rightarrow \mathbb{R}_{+} \cup\{0\}$ and $y: V \rightarrow \mathbb{R}_{+} \cup\{0\}$ an assignment even if $(x, y)$ is potentially infeasible for LP1. In other words initially we have a feasible fractional solution, in the end we will obtain a feasible integral solution, although during the execution of our rounding algorithm an assignment $(x, y)$ is not required to be feasible. Furthermore without loss of generality we assume that for a vertex $v$ with $L(v)=0$ we have $y_{v}=0$.

We need to show that there exists a constant $\delta$ such that if for a connected component LP1 has a feasible solution, then one can (in polynomial time) find an integral feasible solution for $G^{\delta}$.

Definition 9 ( $\delta$-feasible solution). An assignment is called $\delta$-feasible if it is feasible for the graph $G^{\delta}$.

Note that the only difference between LP1's for the graphs $G$ and $G^{\delta}$ is constraint (6).

Definition $10\left(\operatorname{radius}_{(x, y)}\right)$. For a $\delta$-feasible solution $(x, y)$ to LP1 we define a function $\operatorname{radius}_{(x, y)}$ : $V \rightarrow\{0, \ldots, \delta\}$ which for a vertex $u$ assigns the greatest integer $i$ such that there exists a vertex $v$ with $\operatorname{dist}_{G}(v, u)=i$ and $x_{u, v}>0$ (if no such $i$ exists then $\operatorname{radius}_{(x, y)}(u)=0$ ).

We give a brief overview of the rounding algorithm described in subsequent subsection of Section 2. Initially we start with a 1-feasible (fractional) solution $(x, y)$ to LP1 and our goal is to make it integral. We perform several steps where in each step we get more structure on the $\delta$-feasible solution but at the same time the value of $\delta$ will increase. 
In Sections 2.1 2.4 in four non-trivial steps we round the $y$-values of a feasible solution. First, in Section 2.1 we define a caterpillar structure which is a key structure in the rounding process. We show that in polynomial time we can find a 5 -feasible solution together with a caterpillar structure $\left(P, P^{\prime}\right)$ such that all vertices outside of the caterpillar structure have integral $y$-values. In Section 2.2 we define the $y$-flow and chain shifting operations which allow for transferring $y$-values between distant vertices using intermediate vertices on the caterpillar structure. Unfortunately, because the capacities are non-uniform and hard, to find a rounding flow for a caterpillar structure we need more assumptions. To overcome this difficulty in the most challenging part of the rounding process, that is in Section 2.3, we define a safe caterpillar structure and show how to split a given caterpillar structure into a set of safe caterpillar structures (at the cost of increasing radius of the $\delta$-feasible solution). In Section 2.4 we design a rounding procedure for a safe caterpillar structure, obtaining a $c$-feasible solution with integral $y$-values, for some constant $c$. We would like to note, that for uniform capacities every caterpillar structure is safe, therefore for non-uniform capacities we have to design much more involved tools comparing to the previously known uniform capacities case.

Finally in Section 2.5 we show, that using standard techniques, when we have integral $y$-values then rounding $x$-values is simple, obtaining a constant factor approximation algorithm.

\section{LP rounding for hard-capacities}

\subsection{Group shifting and caterpillar structure}

In the first phase of our procedure we obtain a path-like structure containing all vertices with non-integral $y$-values. We first define the notion of shifting values between variables of LP1 relaxation.

Definition 11 (shifting). For an assignment $(x, y)$ for the $L P$, two distinct vertices $a, b \in V$ and a positive real $\alpha \leq \min \left(y_{a}, 1-y_{b}\right)$ such that $L(a) \leq L(b)$ by shifting $\alpha$ from $a$ to $b$ we consider the following operation:

1. Let $\epsilon=\frac{\alpha}{y_{a}}$; for each $v \in V$ let $\Delta_{v}=\epsilon x_{a, v}$, decrease $x_{a, v}$ by $\Delta_{v}$ and increase $x_{b, v}$ by $\Delta_{v}$.

2. Increase $y_{b}$ by $\alpha$, and decrease $y_{a}$ by $\alpha$.

Lemma 12. Let $(x, y)$ be a $\delta$-feasible solution to LP. Let $\left(x^{\prime}, y^{\prime}\right)$ be a result of shifting $\alpha$ from a to $b$, for some $\alpha, a, b$ such that $L(a) \leq L(b), 0<\alpha \leq \min \left(y_{a}, 1-y_{b}\right)$. Then $\left(x^{\prime}, y^{\prime}\right)$ is a $\left(\delta+\operatorname{dist}_{G}(a, b)\right)$-feasible solution and for each vertex $v \neq b$ we have $\operatorname{radius}_{\left(x^{\prime}, y^{\prime}\right)}(v) \leq \operatorname{radius}_{(x, y)}(v)$ whereas radius $_{\left(x^{\prime}, y^{\prime}\right)}(b) \leq$ $\max \left(\operatorname{radius}_{(x, y)}(a)+\operatorname{dist}_{G}(a, b), \operatorname{radius}_{(x, y)}(b)\right)$.

Proof. First we prove that $\left(x^{\prime}, y^{\prime}\right)$ is a $\left(\delta+\operatorname{dist}_{G}(a, b)\right)$-feasible solution. Since the sum of all $y$-values in $(x, y)$ is equal to the sum of all $y$-values in $\left(x^{\prime}, y^{\prime}\right)$, constraint (1) of LP1 is satisfied. To prove (2) of LP1, it is enough to consider the variables $x_{a, v}^{\prime}, x_{b, v}^{\prime}$ for each vertex $v$, that is:

$$
\begin{aligned}
& x_{b, v}^{\prime}=x_{b, v}+\epsilon x_{a, v} \leq y_{b}+\epsilon y_{a}=y_{b}+\alpha=y_{b}^{\prime} \\
& x_{a, v}^{\prime}=x_{a, v}-\epsilon x_{a, v} \leq y_{a}(1-\epsilon)=y_{a}^{\prime} .
\end{aligned}
$$

For (3) of LP1, we only verify $v=a$ and $v=b$ since for other vertices the sum did not change.

$$
\begin{aligned}
\sum_{v \in V} x_{b, v}^{\prime} & =\sum_{v \in V}\left(x_{b, v}+\epsilon x_{a, v}\right)=\left(\sum_{v \in V} x_{b, v}\right)+\epsilon\left(\sum_{v \in V} x_{a, v}\right) \leq L(b) y_{b}+\epsilon L(a) y_{a} \leq L(b) y_{b}+\epsilon L(b) y_{a} \\
& =L(b)\left(y_{b}+\epsilon y_{a}\right)=L(b) y_{b}^{\prime} \\
\sum_{v \in V} x_{a, v}^{\prime} & =\sum_{v \in V}\left(x_{a, v}-\epsilon x_{a, v}\right)=(1-\epsilon) \sum_{v \in V} x_{a, v} \leq(1-\epsilon) L(a) y_{a}=L(a) y_{a}^{\prime}
\end{aligned}
$$


For each vertex $u$ the sum $\sum_{v \in V} x_{v, u}$ is equal to $\sum_{v \in V} x_{v, u}^{\prime}$ hence constraint (4) is satisfied. Constraints (5), (7) may be checked directly, since $\alpha \leq \min \left(y_{a}, 1-y_{b}\right)$.

Since for each vertex $v \neq b$ a variable $x_{v, u}$ can only be decreased (when $v=a$ ), therefore $\operatorname{radius}_{\left(x^{\prime}, y^{\prime}\right)}(v) \leq$ $\operatorname{radius}_{(x, y)}(v)$. For $v=b$ the new radius may increase but it will not exceed $\operatorname{radius}_{(x, y)}(a)+\operatorname{dist}_{G}(a, b)$ since if $x_{b, u}^{\prime}>0$ then either $x_{b, u}>0$ or $x_{a, u}>0$. Consequently constraint (6) is satisfied for $G^{\delta^{\prime}}$ where $\delta^{\prime}=\delta+\operatorname{dist}_{G}(a, b)$ and the lemma follows.

Definition 13 (group shifting). For a $\delta$-feasible solution $(x, y)$ and a set $V_{0} \subseteq V$ by a group shifting we denote the following operation. Assume $V_{0}=\left\{v_{1}, \ldots, v_{\ell}\right\}$, where $L\left(v_{i}\right) \leq L\left(v_{i+1}\right)$ for $1 \leq i<\ell$. As long as there are at least two vertices in $V_{0}$ with fractional $y$-values, let $a$ be the smallest, and $b$ the greatest integer such that $v_{a}, v_{b} \in V_{0}$ are vertices with fractional $y$-values. Shift $\min \left(y_{a}, 1-y_{b}\right)$ from $a$ to $b$.

Lemma 14. Let $(x, y)$ be a $\delta$-feasible solution, $V_{0}$ be a subset of $V$ and $d=\max _{a, b \in V_{0}} \operatorname{dist}_{G}(a, b)$. After group shifting on $V_{0}$ we obtain a $(\delta+d)$-feasible solution $\left(x^{\prime}, y^{\prime}\right)$, where there is at most one vertex in $V_{0}$ with fractional $y$-value and moreover for $v \in V \backslash V_{0}$ we have $\operatorname{radius}_{\left(x^{\prime}, y^{\prime}\right)}(v) \leq \operatorname{radius}_{(x, y)}(v)$.

To make a graph Hamiltonian we use the following lemma known from 1960 [21, 29].

Lemma 15. For any undirected connected graph $G$ there always exists a Hamiltonian path in $G^{3}$ and one can find it in polynomial time.

We define a caterpillar structure which is one of the key ingredients of our rounding process. Intuitively we want to define an auxiliary path-like tree, where adjacent vertices are close in the original graph $G$, vertices with fractional $y$-values are leaves of the tree, and all non-leaf vertices have $y$-values equal to 1 .

Definition 16 (caterpillar structure). By a $\delta$-caterpillar structure for an assignment $(x, y)$ we denote a sequence of distinct vertices $P=\left(v_{1}, \ldots, v_{p}\right)$ together with a sequence $P^{\prime}=\left(v_{0}^{\prime}, \ldots, v_{p+1}^{\prime}\right)$ where:

1. for each $i=1, \ldots, p$ we have $y_{v_{i}}=1$,

2. for each $i=1, \ldots, p-1$ we have $\operatorname{dist}_{G}\left(v_{i}, v_{i+1}\right) \leq \delta$,

3. for each $i=0, \ldots, p+1$ either $v_{i}^{\prime}=$ nil or $v_{i}^{\prime} \in V \backslash\left\{v_{j}: j=1, \ldots, p\right\}$,

4. for each $i=1, \ldots, p$ if $v_{i}^{\prime} \neq$ nil then $L\left(v_{i}\right) \geq L\left(v_{i}^{\prime}\right), 0<y_{v_{i}^{\prime}}<1, \operatorname{dist}_{G}\left(v_{i}, v_{i}^{\prime}\right) \leq \delta$,

5. if $v_{0}^{\prime} \neq$ nil then $\operatorname{dist}_{G}\left(v_{0}^{\prime}, v_{1}\right) \leq \delta, 0<y_{v_{0}^{\prime}}<1$,

6. if $v_{p+1}^{\prime} \neq$ nil then $\operatorname{dist}_{G}\left(v_{p+1}^{\prime}, v_{p}\right) \leq \delta, 0<y_{v_{p+1}^{\prime}}<1$,

7. for each $0 \leq i<j \leq p+1$ if $v_{i}^{\prime} \neq$ nil and $v_{j}^{\prime} \neq$ nil then $v_{i}^{\prime} \neq v_{j}^{\prime}$,

8. $\sum_{v \in V\left(P^{\prime}\right)} y_{v}$ is integral.

We sometimes omit $\delta$ and simply write "caterpillar structure" when the value of $\delta$ is irrelevant.

Lemma 17. For a given feasible LP solution $(x, y)$ we can find a 5-feasible solution $\left(x^{\prime}, y^{\prime}\right)$ together with a 21-caterpillar structure $\left(P, P^{\prime}\right)$ such that each vertex $v \in V \backslash\left(V(P) \cup V\left(P^{\prime}\right)\right)$ has an integral y-value in $\left(x^{\prime}, y^{\prime}\right)$, and the first and last element of the sequence $P^{\prime}$ equals nil.

Proof. Consider the following algorithm for constructing sets $S, S^{\prime}$ and a function $\Phi: V \rightarrow S^{\prime}$. The set $S$ will be an inclusionwise maximal independent set in $G^{2}$ and moreover we ensure that $L(\Phi(v)) \geq L(v)$, for any $v \in V$. 


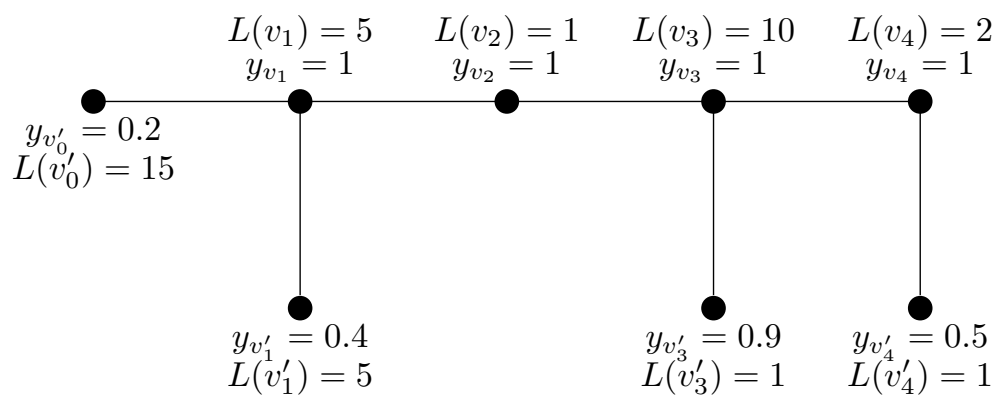

Figure 2: Example of a $\delta$-caterpillar structure $\left(\left(v_{1}, v_{2}, v_{3}, v_{4}\right),\left(v_{0}^{\prime}, v_{1}^{\prime}\right.\right.$, nil, $v_{3}^{\prime}, v_{4}^{\prime}$, nil $\left.)\right)$. Vertices connected by edges are within distance $\delta$ in the graph $G$. Note that the sum of $y$-values over all vertices is integral.

1. Set $V_{0}:=V$ and $S:=S^{\prime}:=\emptyset$.

2. As long as $V_{0} \neq \emptyset$ let $v$ be a highest capacity vertex in $V_{0}$.

- Let $f(v)$ be a highest capacity vertex in $N_{G}[v]$ (potentially $f(v) \notin V_{0}$ ).

- Add $f(v)$ to $S^{\prime}$ and for each $u \in N_{G}\left[N_{G}[v]\right] \cap V_{0}$ set $\Phi(u)=f(v)$.

- Add $v$ to $S$ and set $V_{0}:=V_{0} \backslash N_{G}\left[N_{G}[v]\right]$.

Observe that each time we remove from the set $V_{0}$ all vertices that are within distance two from $v$, hence the set $S$ is an inclusion maximal independent set in $G^{2}$. For this reason vertices in the set $S$ have disjoint neighborhoods and moreover by constraints (4) and (2) of the LP1 we infer that for each $v \in V$ we have:

$$
\sum_{u \in N[v]} y_{u} \geq \sum_{u \in N[v]} x_{u, v}=1
$$

We perform shifting operations to make sure all vertices in the set $S^{\prime}$ have $y$-value equal to one. Consider a vertex $v \in S$ and the corresponding vertex $f(v)$ chosen by the algorithm. As long as $y_{f(v)}<1$ take any $u \in$ $N[v], u \neq f(v)$ such that $y_{u}>0$ and $\operatorname{shift} \min \left(y_{u}, 1-y_{f(v)}\right)$ from $u$ to $f(v)$. Note that $L(u) \leq L(f(v))$ by the definition of $f(v)$ and for this reason shifting is possible. By Lemma 12 after all the shifting operations we have a 3 -feasible solution $(x, y)$, since before a shift from $u$ to $f(v)$ we have $\operatorname{radius}_{(x, y)}(u) \leq 1$, $\operatorname{radius}_{(x, y)}(f(v)) \leq 3$ and $\operatorname{dist}_{G}(u, f(v)) \leq 2$. Moreover by Inequality 8 , we infer, that all the vertices in the set $S^{\prime}$ have $y$-value equal to one, since otherwise a shifting operation from some $u \in N[v]$ to $f(v)$ would be possible.

Observe that by the maximality of the independent set $S$ in $G^{2}$ the graph $G^{5}[S]$ is connected, otherwise we could add a vertex to $S$ still obtaining an independent set in $G^{2}$. Moreover for any two adjacent vertices $u, v \in S$ in $G^{5}[S]$, the vertices $f(u), f(v)$ are adjacent in $G^{7}\left[S^{\prime}\right]$. By the connectivity of $G^{5}[S]$, the graph $G^{7}\left[S^{\prime}\right]$ is also connected. By Lemma 15 we can in polynomial time order the vertices of $S^{\prime}$ to obtain a Hamiltonian path $P$ in $G^{21}\left[S^{\prime}\right]$.

Currently for each vertex $v$ from the set $V \backslash S^{\prime}$ we have $\operatorname{radius}_{(x, y)}(v) \leq 1$. For each $v \in S$ we use group shifting on the set $\Phi^{-1}(f(v)) \backslash S^{\prime}$. Since

$$
\max _{a, b \in \Phi^{-1}(f(v)) \backslash S^{\prime}} \operatorname{dist}_{G}(a, b) \leq \max _{a, b \in \Phi^{-1}(f(v)) \backslash S^{\prime}} \operatorname{dist}_{G}(a, v)+\operatorname{dist}_{G}(v, b) \leq 4,
$$

by Lemma 14 we obtain a 5 -feasible solution $(x, y)$ such that all vertices in the set $S^{\prime}$ have $y$-value equal to one and moreover for each $f(v) \in S^{\prime}$ the set $\Phi^{-1}(f(v)) \backslash S^{\prime}$ contains at most one vertex with fractional 
$y$-value. Let us assume that the already constructed path $P$ is of the form $P=\left(v_{1}, \ldots, v_{p}\right)$. We construct a sequence $P^{\prime}=\left(\right.$ nil $, v_{1}^{\prime}, \ldots, v_{p}^{\prime}$, nil) where as $v_{i}^{\prime}$ we take the only vertex from $\Phi^{-1}\left(v_{i}\right) \backslash S^{\prime}$ that has fractional $y$-value, or we set $v_{i}^{\prime}:=$ nil if $\Phi^{-1}\left(v_{i}\right) \backslash S^{\prime}$ has no vertices with fractional $y$-value. Note that since the way we select vertices to the sets $S, S^{\prime}$ is capacity driven (recall as $v$ we select the highest capacity vertex in $V_{0}$ and as $f(v)$ we select a highest capacity vertex in $N[v])$, for each vertex $u \in \Phi^{-1}\left(v_{i}\right)$ we have $L(u) \leq L\left(v_{i}\right)$. In this way we have constructed a 5 -feasible solution $(x, y)$ together with a desired 21-caterpillar structure $\left(P, P^{\prime}\right)$.

As the reader might notice in the above proof we always construct a caterpillar structure with $v_{0}^{\prime}=$ $v_{p+1}^{\prime}=$ nil. The reason why the definition of a caterpillar structure allows for $v_{0}^{\prime}$ and $v_{p+1}^{\prime}$ have non-nil values is that in Section 2.3 we will split a caterpillar structure into two smaller pieces and in order to have those pieces satisfy Definition 16 we need $v_{0}^{\prime}$ and $v_{p+1}^{\prime}$.

\section{$2.2 y$-flow and chain shifting}

In the previous section we defined a group shifting operation. Unfortunately we can only perform such an operation if vertices are close. In this section we define notions of $y$-flow and chain shifting which allow us to transfer $y$-value between distant vertices. We will use those tools in Sections 2.3 and 2.4.

Definition 18 (y-flow). For a given assignment $(x, y)$ let $S \subseteq V$ and $T \subseteq V$ be two disjoint sets and let $\mathcal{F}$ be a set containing sequences of the form $\left(\alpha, v_{1}, \ldots, v_{t}\right)$ representing paths, where $\alpha$ is a positive real, each $v_{i} \in V$ is a vertex (for $i=1, \ldots, t$ ), $v_{1} \in S, v_{t} \in T, L\left(v_{1}\right) \leq L\left(v_{t}\right)$ and for $i=2, \ldots, t-1$ we have $v_{i} \notin S \cup T, y_{v_{i}}=1, L\left(v_{i}\right) \geq L\left(v_{1}\right)$. We call $\left(\alpha, v_{1}, \ldots, v_{t}\right)$ a path transferring $\alpha$ from $v_{1}$ to $v_{t}$ through $v_{2}, \ldots, v_{t-1}$. We denote $v_{2}, \ldots, v_{t-1}$ as internal vertices of the path $\left(\alpha, v_{1}, \ldots, v_{t}\right)$.

The set $\mathcal{F}$ is a $y$-flow from $S$ to $T$ iff:

- for each $v \in S$ the sum of values transferred from $v$ in $\mathcal{F}$ is at most $y_{v}$,

- for each $v \in T$ the sum of values transferred to $v$ in $\mathcal{F}$ is at most $1-y_{v}$,

- for each $v \in V \backslash(S \cup T)$ the sum of values transferred through $v$ in $\mathcal{F}$ is at most 1 .

For a given $y$-flow $\mathcal{F}$ from $S$ to $T$ we define $G_{\mathcal{F}}=(V, A)$ as an auxiliary directed graph with the same vertex set as $G$, where an $\operatorname{arc}(u, v)$ belongs to $A$ iff there is a path in $\mathcal{F}$ containing $u$ and $v$ as consecutive vertices in exactly this order. We call the $y$-flow $\mathcal{F}$ acyclic iff the directed flow graph $G_{\mathcal{F}}$ is acyclic. Furthermore we define a function $f_{\mathcal{F}}: A \rightarrow(0,1]$, which for an $\operatorname{arc}(u, v)$ assigns the sum of $\alpha$ values in all the paths in $\mathcal{F}$ that contain $u$ and $v$ as consecutive vertices. Moreover by $f l_{\mathcal{F}}: A \rightarrow \mathbb{R}_{+}$we denote a function, which for an arc $(u, v)$ assigns the sum of terms $L(s) \alpha$ over all paths from $\mathcal{F}$ that start with $\alpha$ and $s \in S$ and contain $u, v$ as consecutive elements. Intuitively by $f_{\mathcal{F}}((u, v))$ we denote the fractional number of centers that are transferred from $u$ to $v$, whereas by $f l_{\mathcal{F}}((u, v))$ we denote the fractional number of vertices (clients) that were previously covered by $u$ and will be covered by $v$ after the shifting operation (see Fig. 3).

Now we show that if we are given an acyclic $y$-flow $\mathcal{F}$ then we can transfer $y$-values using a chain shifting method without increasing the radius of vertices by too much. Formal definitions and lemmas follow.

Definition 19 (chain shifting). Let $\mathcal{F}$ be an acyclic $y$-flow from $S$ to $T$ and let $(x, y)$ be a $\delta$-feasible solution. Let $G_{\mathcal{F}}=(V, A)$ be the auxiliary acyclic flow graph.

By chain shifting we denote the following operation:

- For each $u, v \in V$, set $\Delta_{u, v}=0$. 


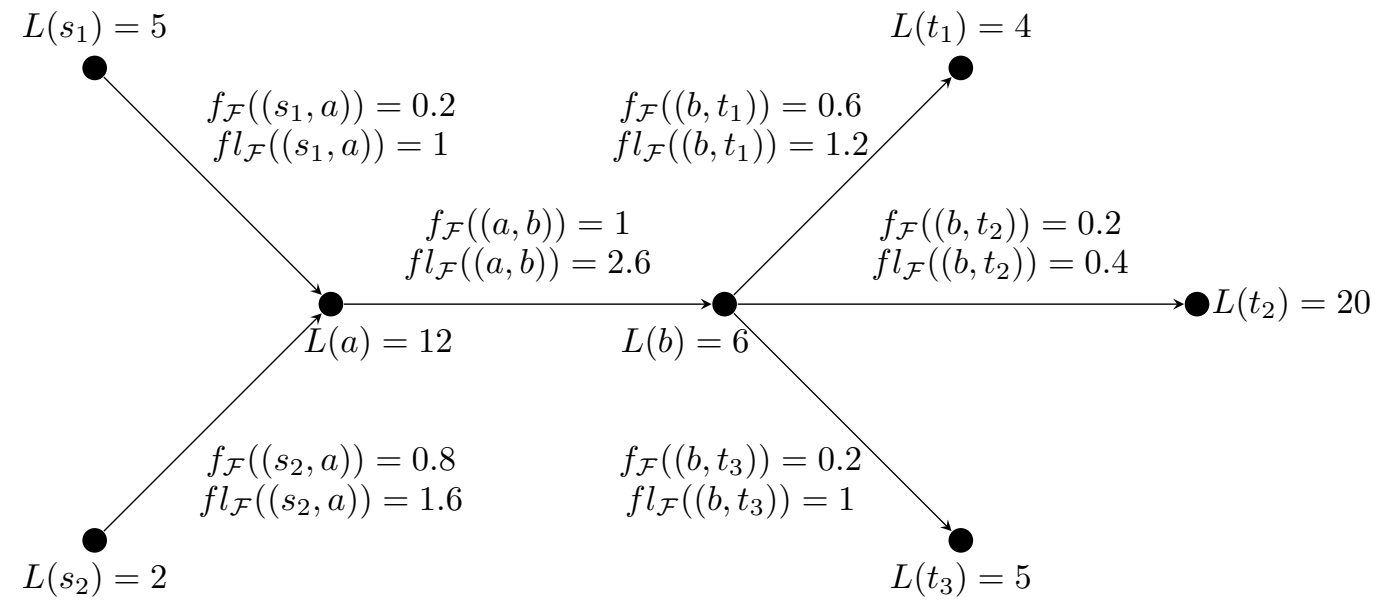

Figure 3: The graph $G_{\mathcal{F}}$ for an acyclic $y$-flow $\mathcal{F}=\left\{\left(0.2, s_{1}, a, b, t_{3}\right),\left(0.6, s_{2}, a, b, t_{1}\right),\left(0.2, s_{2}, a, b, t_{2}\right)\right\}$ from $S=\left\{s_{1}, s_{2}\right\}$ to $T=\left\{t_{1}, t_{2}, t_{3}\right\}$, where $y_{s_{1}}=0.4, y_{s_{2}}=y_{a}=y_{b}=1, y_{t_{1}}=0, y_{t_{2}}=0.8, y_{t_{3}}=0.1$. Note that even though each path in $\mathcal{F}$ has starting point capacity not greater than its ending point capacity the vertex $t_{1} \in T$ is reachable from $s_{1} \in S$ in $G_{\mathcal{F}}$ despite the fact that $L\left(s_{1}\right)>L\left(t_{1}\right)$.

- For each $\operatorname{arc}(u, a) \in A$ in reverse topological ordering of $G_{\mathcal{F}}$ :

- For each $v \in V$, let $\Delta=x_{u, v} f l_{\mathcal{F}}(u, a) /\left(L(u) y_{u}\right)$, set $\Delta_{a, v}=\Delta_{a, v}+\Delta$ and $\Delta_{u, v}=\Delta_{u, v}-\Delta$.

- For each $u, v \in V$, set $x_{u, v}=x_{u, v}+\Delta_{u, v}$.

- For each $s \in S$ decrease $y_{s}$ by $\sum_{(s, u) \in A} f_{\mathcal{F}}((s, u))$.

- For each $t \in T$ increase $y_{t}$ by $\sum_{(u, t) \in A} f_{\mathcal{F}}((u, t))$.

For a directed graph $G=(V, A)$, for a vertex $v$, we denote $N^{i n}(v)=\{u:(u, v) \in A\}$ and $N^{\text {out }}(v)=$ $\{u:(v, u) \in A\}$.

Lemma 20. Let $\left(x^{\prime}, y^{\prime}\right)$ be the result of the chain shifting operation on a $\delta$-feasible solution $(x, y)$ according to an acyclic $y$-flow $\mathcal{F}$ from $S$ to $T$. If $d$ is the greatest distance in $G$ between two adjacent vertices in $G_{\mathcal{F}}$, then $\left(x^{\prime}, y^{\prime}\right)$ is a $(\delta+d)$-feasible solution, and for each vertex $v$ of indegree zero in $G_{\mathcal{F}}$, we have $\operatorname{radius}_{\left(x^{\prime}, y^{\prime}\right)}(v) \leq \operatorname{radius}_{(x, y)}(v)$, whereas for other vertices $v$, we have

$$
\operatorname{radius}_{\left(x^{\prime}, y^{\prime}\right)}(v) \leq \max \left(\operatorname{radius}_{(x, y)}(v), \underset{a \in N_{G_{\mathcal{F}}}^{i n}(v)}{\max }\left(\operatorname{radius}_{(x, y)}(a)+\operatorname{dist}_{G}(a, v)\right)\right) .
$$

Furthermore for each $v \in V \backslash(S \cup T)$ its $y$-value is the same in $(x, y)$ and $\left(x^{\prime}, y^{\prime}\right)$.

Proof. First observe that for any $\operatorname{arc}(u, v)$ in $G_{\mathcal{F}}$ we have the following inequality

$$
f l_{\mathcal{F}}(u, v) / L(u) \leq f_{\mathcal{F}}(u, v),
$$

since on each path in $\mathcal{F}$ the starting vertex has a smallest capacity. Moreover by the definition of a $y$-flow, through each vertex $u \in V$ at most $y_{u}$ units are transferred, hence by Inequality (9) we have:

$$
\sum_{a \in N_{G_{\mathcal{F}}}^{o u t}(u)} f l_{\mathcal{F}}(u, a) / L(u) \leq \sum_{a \in N_{G_{\mathcal{F}}}^{\text {out }}(u)} f_{\mathcal{F}}(u, a) \leq y_{u} \text { and }
$$




$$
\left(1-\sum_{a \in N_{G_{\mathcal{F}}}^{\text {out }}(u)} \frac{f l_{\mathcal{F}}(u, a)}{L(u) y_{u}}\right) \geq 0 .
$$

We want to show that $\left(x^{\prime}, y^{\prime}\right)$ is a $(\delta+d)$-feasible solution. Observe that by the definition of chain shifting $\sum_{v \in V} y_{v}^{\prime}=\sum_{v \in V} y_{v}$, and for each vertex $u \in V$ we have $\sum_{v \in V} x_{v, u}^{\prime}=\sum_{v \in V} x_{v, u}$. Therefore constraints (1) and (4) are satisfied. Now we check constraint (7). Observe that for any $u, v \in V$ we have:

$$
x_{u, v}^{\prime}=x_{u, v}+\Delta_{u, v} \geq x_{u, v}-\sum_{a \in N_{G_{\mathcal{F}}}^{\text {out }}(u)} x_{u, v} f l_{\mathcal{F}}(u, a) /\left(L(u) y_{u}\right)
$$

(by (10) $) \geq x_{u_{v}}-x_{u, v} y_{u} / y_{u}=0$.

Next we check constraint (2). Consider any $u, v \in V$. Observe that if $u \in V \backslash(S \cup T)$ then either $x_{u, v}^{\prime}=x_{u, v} \leq y_{u}=y_{u}^{\prime}$ (which happens if $u$ does not belong to any path in $\mathcal{F}$ ) or $y_{u}^{\prime}=1 \geq x_{u, v}^{\prime}$ (when $u$ is an internal vertex in a path from $\mathcal{F})$. On the other hand for $s \in S, v \in V$ we have $x_{s, v} \leq y_{s}$ and hence by Inequality (9):

$$
x_{s, v}^{\prime}=x_{s, v}\left(1-\sum_{a \in N_{G_{\mathcal{F}}}^{\text {out }}(s)} \frac{f l_{\mathcal{F}}(s, a)}{L(s) y_{s}}\right) \leq y_{s}\left(1-\sum_{a \in N_{G_{\mathcal{F}}}^{\text {out }}(s)} \frac{f_{\mathcal{F}}(s, a)}{y_{s}}\right)=y_{s}^{\prime} .
$$

Similarly for $t \in T, v \in V$ we have

$$
x_{t, v}^{\prime}=x_{t, v}+\sum_{a \in N_{G_{\mathcal{F}}}^{i n}(t)} \frac{f l_{\mathcal{F}}(a, t) x_{a, v}}{L(a) y_{a}} \leq y_{t}+\sum_{a \in N_{G_{\mathcal{F}}}^{i n}(t)} \frac{f l_{\mathcal{F}}(a, t)}{L(a)} \leq y_{t}+\sum_{a \in N_{G_{\mathcal{F}}}^{i n}(t)} f_{\mathcal{F}}(a, t)=y_{t}^{\prime} .
$$

As for constraint (3) for a vertex $u \in V$ we have:

$$
\begin{aligned}
& \sum_{v \in V} x_{u, v}^{\prime}=\sum_{v \in V} x_{u, v}+\sum_{v \in V} \sum_{a \in N_{G_{\mathcal{F}}}^{\text {in }}(u)} \frac{f l_{\mathcal{F}}(a, u) x_{a, v}}{L(a) y_{a}}-\sum_{v \in V} \sum_{a \in N_{G_{\mathcal{F}}}^{\text {out }}(u)} \frac{f l_{\mathcal{F}}(u, a) x_{u, v}}{L(u) y_{u}} \\
&=\sum_{v \in V} x_{u, v}\left(1-\sum_{a \in N_{G_{\mathcal{F}}}^{\text {out }}(u)} \frac{f l_{\mathcal{F}}(u, a)}{L(u) y_{u}}\right)+\sum_{v \in V} \sum_{a \in N_{G_{\mathcal{F}}}^{i n}(u)} \frac{f l_{\mathcal{F}}(a, u) x_{a, v}}{L(a) y_{a}} \\
&\text { (by (3) of LP1 and (11) }) \leq L(u) y_{u}\left(1-\sum_{a \in N_{G_{\mathcal{F}}}^{\text {out }}(u)} \frac{f l_{\mathcal{F}}(u, a)}{L(u) y_{u}}\right)+\sum_{a \in N_{G_{\mathcal{F}}}^{i n}(u)} \sum_{v \in V} \frac{f l_{\mathcal{F}}(a, u) x_{a, v}}{L(a) y_{a}} \\
&=L(u) y_{u}-\sum_{a \in N_{G_{\mathcal{F}}}^{\text {out }}(u)} f l_{\mathcal{F}}(u, a)+\sum_{a \in N_{G_{\mathcal{F}}}^{i n}(u)} f l_{\mathcal{F}}(a, u) /\left(L(a) y_{a}\right) \sum_{v \in V} x_{a, v} \\
& \text { (by (3) of LP1) } \leq L(u) y_{u}-\sum_{a \in N_{G_{\mathcal{F}}}^{\text {out }}(u)} f l_{\mathcal{F}}(u, a)+\sum_{a \in N_{G_{\mathcal{F}}}^{i n}(u)} f l_{\mathcal{F}}(a, u) .
\end{aligned}
$$

Since for $u \in V \backslash(S \cup T)$ by the definition of $y$-flow $\sum_{a \in N_{G_{\mathcal{F}}}^{\text {out }}(u)} f l_{\mathcal{F}}(u, a)=\sum_{a \in N_{G_{\mathcal{F}}}^{\text {in }}(u)} f l_{\mathcal{F}}(a, u)$ we infer $\sum_{v \in V} x_{u, v}^{\prime} \leq L(u) y_{u}=L(u) y_{u}^{\prime}$. For $s \in S$ we have $N_{G_{\mathcal{F}}}^{i n}(s)=\emptyset$ and $f l_{\mathcal{F}}(s, a)=f_{\mathcal{F}}(s, a) L(s)$ hence $\sum_{v \in V} x_{s, v}^{\prime} \leq L(s)\left(y_{s}-\sum_{a \in N_{G_{\mathcal{F}}}^{\text {out }}(s)} f_{\mathcal{F}}(s, a)\right)=L(s) y_{s}^{\prime}$. Similarly for $t \in T$ we have $N_{G_{\mathcal{F}}}^{\text {out }}(s)=\emptyset$ 
and $f l_{\mathcal{F}}(a, t) \geq f_{\mathcal{F}}(a, t) L(t)$ hence $\sum_{v \in V} x_{s, v}^{\prime} \leq L(t)\left(y_{s}+\sum_{a \in N_{G_{\mathcal{F}}}^{i n}(t)} f_{\mathcal{F}}(a, t)\right)=L(t) y_{t}^{\prime}$. Therefore constraint (3) is satisfied.

Constraint (5) may be checked directly by the definition of $y$-flow. To check the radius of each vertex in $\left(x^{\prime}, y^{\prime}\right)$ (that is to verify $(\delta+d)$-feasibility of constraint (6) we observe that if $x_{u, v}^{\prime}>0$ then either $x_{u, v}>0$ or $x_{a, v}>0$ for some $a \in N_{G_{\mathcal{F}}}^{i n}(v)$.

\subsection{Separable caterpillar structure}

If we knew that in the caterpillar structure $\left(P, P^{\prime}\right)$ produced by Lemma 17 the capacity of each vertex in $P$ is not smaller than the capacity of each vertex in $P^{\prime}$ then we could skip this section. Unfortunately some vertices of $V(P)$ may have smaller capacity than some vertices of $V\left(P^{\prime}\right)$ and for this reason we define the notion of dangerous, safe and separable caterpillar structures.

Definition 21 (safe, dangerous). For a caterpillar structure $\mathcal{P}=\left(P=\left(v_{1}, \ldots, v_{p}\right),\left(v_{0}^{\prime}, \ldots, v_{p+1}^{\prime}\right)\right)$, by $\Gamma(\mathcal{P}) \subseteq V(P)$ we denote the set containing all vertices $v_{i}$, such that there exist $0 \leq i_{0}<i<i_{1} \leq p+1$, such that $v_{i_{0}}^{\prime} \neq$ nil, $L\left(v_{i_{0}}^{\prime}\right)>L\left(v_{i}\right)$ and $v_{i_{1}}^{\prime} \neq$ nil, $L\left(v_{i_{1}}^{\prime}\right)>L\left(v_{i}\right)$.

A caterpillar structure $\mathcal{P}$ is safe if $\Gamma(\mathcal{P})=\emptyset$ and dangerous otherwise.

Definition 22 (separable). Let $(x, y)$ be a $\delta$-feasible solution and let $\mathcal{P}=\left(P=\left(v_{1}, \ldots, v_{p}\right)\right.$, $\left.P^{\prime}=\left(v_{0}^{\prime}, \ldots, v_{p+1}^{\prime}\right)\right)$ be a dangerous caterpillar structure. We call $\mathcal{P}$ separable iff there exists $1 \leq i \leq p$ such that $v_{i} \in \Gamma(\mathcal{P}), L\left(v_{i}\right)=\min _{v \in \Gamma(\mathcal{P})} L(v)$ and either:

- $S_{1} \geq\left\lceil S_{2}\right\rceil-S_{2}$, where $S_{2}=\sum_{\substack{j=i+1, \ldots, p+1 \\ v_{j}^{\prime} \neq \text { nil }}} y_{v_{j}^{\prime}}$ and $S_{1}$ is the sum of values $\left(1-y_{v}\right)$ where $v \in V, v=$ $v_{j}^{\prime}, L(v)>L\left(v_{i}\right)$ for some $i<j \leq p+1$, or,

- $S_{1} \geq\left\lceil S_{2}\right\rceil-S_{2}$, where $S_{2}=\sum_{\substack{j=0, \ldots, i-1 \\ v_{j}^{\prime} \neq \text { nil }}} y_{v_{j}^{\prime}}$ and $S_{1}$ is the sum of values $\left(1-y_{v}\right)$ where $v \in V, v=$ $v_{j}^{\prime}, L(v)>L\left(v_{i}\right)$ for some $0 \leq j<i$.

We call such $i$ as above a witness of separability of $\mathcal{P}$. A caterpillar structure that is not separable is called non-separable.

The intuition behind the sums $S_{1}, S_{2}$ is as follows. The sum $S_{2}$ contains all the $y$-values of vertices of $P^{\prime}$ to the right (or left) of $i$. Since we want to round all the $y$-values of vertices of $P^{\prime}$, if we want to split the caterpillar structure $\left(P, P^{\prime}\right)$ by removing the edge $v_{i} v_{i+1}$ (or $v_{i-1} v_{i}$ ), we need to send $\left\lceil S_{2}\right\rceil-S_{2}$ units of flow to the part that does not contain $v_{i}$, in order to make the sum of $y$-values over all the leaves in both new caterpillar structures integral. That is to satisfy (8) of Definition 16. In $S_{1}$ we sum over all vertices, that can potentially receive flow if we start a path at $v_{i}$, and the value $\left(1-y_{v}\right)$ is the $y$-value a vertex $v$ may receive.

An example of a separable caterpillar structure is depicted in Fig. 4. Observe that a non-separable path structure may be dangerous as in Fig. 5 .

Lemma 23. Let $\mathcal{P}=\left(\left(v_{1}, \ldots, v_{p}\right),\left(v_{0}^{\prime}, \ldots, v_{p+1}^{\prime}\right)\right)$ be a dangerous caterpillar structure and let $i$ be an index such that $v_{i} \in \Gamma(\mathcal{P})$ and $L\left(v_{i}\right)=\min _{v \in \Gamma(\mathcal{P})} L(v)$. Moreover let $j$ be an index such that $v_{j}^{\prime} \neq$ nil, $L\left(v_{j}^{\prime}\right)>L\left(v_{i}\right)$. Then for any $a \in[\min (i, j), \max (i, j)]$ we have $L\left(v_{a}\right) \geq L\left(v_{i}\right)$.

Proof. Assume the contrary, that is assume that such $a$ exists. Clearly $a \neq i$ (since then $L\left(v_{a}\right)=L\left(v_{i}\right)$ ) and also $a \neq j$ because $L\left(v_{j}\right) \geq L\left(v_{j}^{\prime}\right)>L\left(v_{i}\right)$ (where the first inequality follows by 4 of Def. 16. W.1.o.g. assume that $i<j$ (the other case is symmetric). By the definition of $\Gamma$ (Def. 21) we infer that there exists $0 \leq i_{0}<i$ such that $v_{i_{0}}^{\prime} \neq$ nil and $L\left(v_{i_{0}}^{\prime}\right)>L\left(v_{i}\right)>L\left(v_{a}\right)$. Consequently $v_{a} \in \Gamma(\mathcal{P})$ which contradicts the fact that $v_{i}$ is a smallest capacity vertex in $\Gamma(\mathcal{P})$. 
Lemma 24. Let $\mathcal{P}=\left(\left(v_{1}, \ldots, v_{p}\right),\left(v_{0}, \ldots, v_{p+1}\right)\right)$ be a dangerous non-separable caterpillar structure and let $\ell=\min _{v \in \Gamma(\mathcal{P})} L(v)$. For $I=\left\{i: 0 \leq i \leq p+1 \wedge v_{i}^{\prime} \neq\right.$ nil $\left.\wedge L\left(v_{i}^{\prime}\right)>\ell\right\}$ we have $\sum_{i \in I}\left(1-y_{v_{i}^{\prime}}\right)<2$.

Proof. Consider any $v_{i} \in \Gamma(\mathcal{P})$ such that $L\left(v_{i}\right)=\ell$. Let $I_{1}=I \cap[0, i-1]$ and $I_{2}=I \cap[i+1, p+1]$ (note that $I=I_{1} \cup I_{2}$ ). We know that $v_{i}$ is not a witness of separability hence each of the two sums $S_{1}$ in Definition 22 is strictly smaller than 1 , since otherwise we would have $S_{1} \geq 1 \geq\left\lceil S_{2}\right\rceil-S_{2}$. Consequently $\sum_{i \in I_{1}}\left(1-y_{v_{i}^{\prime}}\right)<1$ and similarly $\sum_{i \in I_{2}}\left(1-y_{v_{i}^{\prime}}\right)<1$.

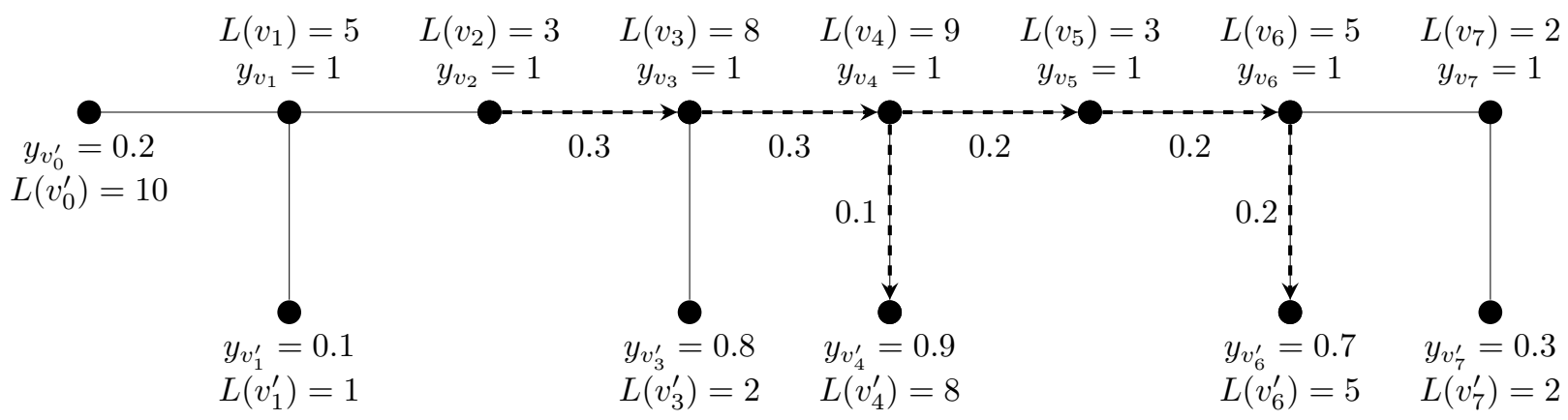

Figure 4: A separable caterpillar structure $\left(P=\left(v_{1}, \ldots, v_{7}\right), P^{\prime}=\left(v_{0}^{\prime}, v_{1}^{\prime}\right.\right.$, nil, $v_{2}^{\prime}, v_{3}^{\prime}$, nil, $v_{5}^{\prime}, v_{6}^{\prime}$, nil, nil $\left.)\right)$, where $\Gamma\left(\left(P, P^{\prime}\right)\right)=\left\{v_{1}, v_{2}, v_{5}\right\}$ (note that $v_{7} \notin \Gamma\left(\left(P, P^{\prime}\right)\right)$, since $v_{8}^{\prime}=$ nil). By dashed edges an acyclic flow $\mathcal{F}=\left\{\left(0.1, v_{2}, v_{3}, v_{4}, v_{4}^{\prime}\right),\left(0.2, v_{2}, v_{3}, v_{4}, v_{5}, v_{6}, v_{6}^{\prime}\right)\right\}$ from $\left\{v_{2}\right\}$ to $\left\{v_{4}^{\prime}, v_{6}^{\prime}\right\}$ is marked with values $f_{\mathcal{F}}$ printed in the middle of each arc.

In the following lemma we use the procedure $\operatorname{SepaRATE}\left(\mathcal{P}=\left(P, P^{\prime}\right)\right)$ which given a $\delta$-caterpillar structure produces a set of non-separable $\delta$-caterpillar structures. The pseudocode of SEPARATE is given as Algorithm 1 and at high level it performs the following steps:

1. (Lines 1-2) If $\left(P, P^{\prime}\right)$ is non-separable then return $\mathcal{P}$.

2. (Line 3) Otherwise let $i$ be a witness from Definition 22 with the smallest value of $L\left(v_{i}\right)$ and assume that $i$ is a witness due to the first conditition in the definition (the other case is symmetric).

3. (Lines 4-5) If the sum $S_{2}$ is integral, then run the procedure recursively on two caterpillar structures $\left(\left(v_{1}, \ldots, v_{i}\right),\left(v_{0}^{\prime}, \ldots, v_{i}^{\prime}\right.\right.$, nil $\left.)\right),\left(\left(v_{i+1}, \ldots, v_{p}\right),\left(\right.\right.$ nil, $\left.\left.v_{i+1}^{\prime}, \ldots, v_{p+1}^{\prime}\right)\right)$ and return the set of obtained non-separable $\delta$-caterpillar structures.

4. (Lines 6-9) Let $I=\left\{j: i<j \leq p+1, v_{j}^{\prime} \neq\right.$ nil, $L\left(v_{j}^{\prime}\right)>L\left(v_{i}\right)$, that is the set of indices used in the definition of the sum $S_{1}$ and denote $I=\left\{i_{1}, \ldots, i_{r}\right\}$, where $i_{1}<\ldots<i_{r}$ and let $\ell_{0}$ be the smallest integer such that $\sum_{j \in I, j \leq \ell_{0}}\left(1-y_{v_{j}^{\prime}}\right) \geq\left\lceil S_{2}\right\rceil-S_{2}$ (such $\ell_{0}$ exists since $i$ is a witness of separability).

5. (Lines 10-11) Construct an acyclic $y$-flow $\mathcal{F}$ from $\left\{v_{i}\right\}$ to $\left\{v_{j}^{\prime}: j \in I, j \leq \ell_{0}\right\}$. That is for each $j \in I, j<\ell_{0}$ add to $\mathcal{F}$ a sequence $\left(1-y_{v_{j}^{\prime}}, v_{i}, v_{i+1}, \ldots, v_{j}, v_{j}^{\prime}\right)$ and for $j=\ell_{0}$ add to $\mathcal{F}$ a sequence $\left(\alpha, v_{i}, v_{i+1}, \ldots, v_{\ell_{0}}, v_{\ell_{0}}^{\prime}\right)$, where $\alpha=\left(\left\lceil S_{2}\right\rceil-S_{2}\right)-\sum_{j \in I, j<\ell_{0}}\left(1-y_{v_{j}^{\prime}}\right)$ (see Fig. 4 for illustration); perform chain shifting according to $\mathcal{F}$.

6. (Lines 12-18) If $i<p$ then run the procedure recursively on the caterpillar structure $\left(\left(v_{i+1}, \ldots, v_{p}\right)\right.$, $\left(\right.$ nil, $\left.v_{i+1}^{\prime \prime}, \ldots, v_{p+1}^{\prime \prime}\right)$ ), where $v_{j}^{\prime \prime}=v_{j}^{\prime}$ if $y_{v_{j}^{\prime}}<1$ and $v_{j}^{\prime \prime}=$ nil otherwise for $i+1 \leq j \leq p+1$ (if $i=p$ we already know that $y_{v_{p+1}^{\prime}}=1$ ). 
7. (Lines 19-21) If $i=1$ then perform group shifting on the set $\left\{v_{0}^{\prime}, v_{1}, v_{1}^{\prime}\right\} \backslash\{$ nil $\}$ and return.

8. (Lines 22-23) If $v_{i}^{\prime} \neq$ nil then $\operatorname{shift} \min \left(y_{v_{i}^{\prime}}, 1-y_{v_{i}}\right)$ from $y_{v_{i}^{\prime}}$ to $y_{v_{i}}$ (by the properties of a caterpillar structure we know that $\left.L\left(v_{i}\right) \geq L\left(v_{i}^{\prime}\right)\right)$.

9. (Lines 24-29) If $y_{v_{i}}=1$ then run the procedure recursively on the caterpillar structure $\left(\left(v_{1}, \ldots, v_{i}\right)\right.$, $\left(v_{0}^{\prime}, \ldots, v_{i-1}^{\prime}, u\right.$, nil $\left.)\right)$, where $u=v_{i}^{\prime}$ if $v_{i}^{\prime} \neq$ nil, $y_{v_{i}^{\prime}}>0$, or $u=$ nil otherwise.

10. (Lines 30-31) Otherwise (if $\left.y_{v_{i}}<1\right)$ run the procedure recursively on the caterpillar structure $\left(\left(v_{1}, \ldots, v_{i-1}\right)\right.$, $\left.\left(v_{0}^{\prime}, \ldots, v_{i-1}^{\prime}, v_{i}\right)\right)$.

Lemma 25. For a given feasible LP solution $(x, y)$ we can find a 68 -feasible solution $\left(x^{\prime}, y^{\prime}\right)$ together with a set of vertex disjoint non-separable 21-caterpillar structures $\mathcal{S}$ such that each vertex $v$ outside of the set has an integral $y$-value in $\left(x^{\prime}, y^{\prime}\right)$. Furthermore for each vertex $v$ that belongs to some caterpillar structure from $\mathcal{S}$ we have $\operatorname{radius}_{\left(x^{\prime}, y^{\prime}\right)}(v) \leq 47$.

Proof. First we use Lemma 17 to obtain a 5 -feasible solution $\left(x^{\prime}, y^{\prime}\right)$ together with a 21-caterpillar structure $\left(P_{0}, P_{0}^{\prime}\right)$ such that each vertex outside $\left(P_{0}, P_{0}^{\prime}\right)$ has an integral $y$-value in $\left(x^{\prime}, y^{\prime}\right)$. Next we run the procedure SEPARATE on the caterpillar structure $\left(P_{0}, P_{0}^{\prime}\right)$ using $\left(x^{\prime}, y^{\prime}\right)$ as the assignment.

Let us prove, that given a $\delta$-caterpillar structure the procedure SEPARATE indeed returns a set of nonseparable $\delta$-caterpillar structures. By Lemma 23 the set $\mathcal{F}$ created in Line 10 of the procedure SEPARATE is indeed an acyclic $y$-flow. Furthermore caterpillar structures created in Lines 5, 18, 29, 31. are indeed $\delta$ caterpillar structures since they satisfy all the conditions of Definition 16. Moreover observe that if a vertex $v$ belongs to $\Gamma\left(\mathcal{P}^{\prime}\right)$, where $\mathcal{P}^{\prime}$ is a caterpillar structure created in one of Lines $5,18,29,31$, then $v \in \Gamma(\mathcal{P})$.

Since some vertices of the caterpillar structure created in Lines 18, 29 and 31 may have increased radius we need to argue why the radius does not grow too much in the subsequent recursive calls.

Let $\mathcal{S}$ be the set of non-separable 21-caterpillar structures, which is a result of $\operatorname{Separate}\left(P_{0}, P_{0}^{\prime}\right)$. Observe that the caterpillar structures are vertex disjoint and moreover each vertex $v$ which does not belong to any caterpillar structure from $S$ has an integral $y$-value in $\left(x^{\prime}, y^{\prime}\right)$.

Due to Lemmas 12 14 20 when we modify the assignment $\left(x^{\prime}, y^{\prime}\right)$ by shifting, group shifting or chain shifting we satisfy all constraints of the LP possibly except (6). To show 68 -feasibility of $\left(x^{\prime}, y^{\prime}\right)$ we prove that for each caterpillar structure $\left(\left(v_{1}, \ldots, v_{p}\right),\left(v_{0}^{\prime}, \ldots, v_{p+1}^{\prime}\right)\right)$ used as an argument of the procedure SEPARATE there exist two indices $0 \leq i_{0} \leq j_{0} \leq p+1$ such that:

- for each $i \in[1, p]$ we have $\operatorname{radius}_{\left(x^{\prime}, y^{\prime}\right)}\left(v_{i}\right) \leq 5+21 \cdot\left[i \leq i_{0}\right]+21 \cdot\left[i \geq j_{0}\right]$,

- for each $i \in[0, p+1]$ if $v_{i}^{\prime} \neq$ nil then $\operatorname{radius}_{\left(x^{\prime}, y^{\prime}\right)}\left(v_{i}^{\prime}\right) \leq 5+21 \cdot\left[i \leq i_{0}\right]+21 \cdot\left[i \geq j_{0}\right]$,

- for each $i \in\left[0, i_{0}\right) \cup\left(j_{0}, p+1\right]$ such that $v_{i}^{\prime} \neq$ nil for any $v \in \Gamma(\mathcal{P})$ we have $L\left(v_{i}^{\prime}\right) \leq L(v)$.

In the formulas above we use the Iverson's bracket notation, that is $[\phi]$ equals 1 when $\phi$ is true, and 0 otherwise. We call a caterpillar structure satisfying the above properties a good caterpillar structure. The initial caterpillar structure $\left(P_{0}, P_{0}^{\prime}\right)$ is good since we can set $i_{0}=0$ and $j_{0}=p+1$. Hence we assume that a caterpillar structure $\left(P, P^{\prime}\right)$ given as an argument to the SEPARATE procedure is good and we want to show that all recursive calls are given good caterpillar structures. If the procedure exits in Line 2 then it is non-separable, thus we assume that $\left(P, P^{\prime}\right)$ is separable and due to the symmetry w.l.o.g. we may assume that $i$ is a witness satisfying the first condition of Definition 22. Clearly caterpillar structures constructed in Line 5 are good caterpillar structures. Now we investigate caterpillar structures constructed in Lines 18, 29 and 31. Observe that due to the definition of a good caterpillar structure we can prove that $i_{0}<i<j_{0}$. Indeed if $i \in\left[1, i_{0}\right]$ then there is no $0 \leq i^{\prime}<i$ with $v_{i}^{\prime} \neq$ nil and $L\left(v_{i}^{\prime}\right)>L\left(v_{i}\right)$ and similarly for $i \in\left[j_{0}, p\right]$ 


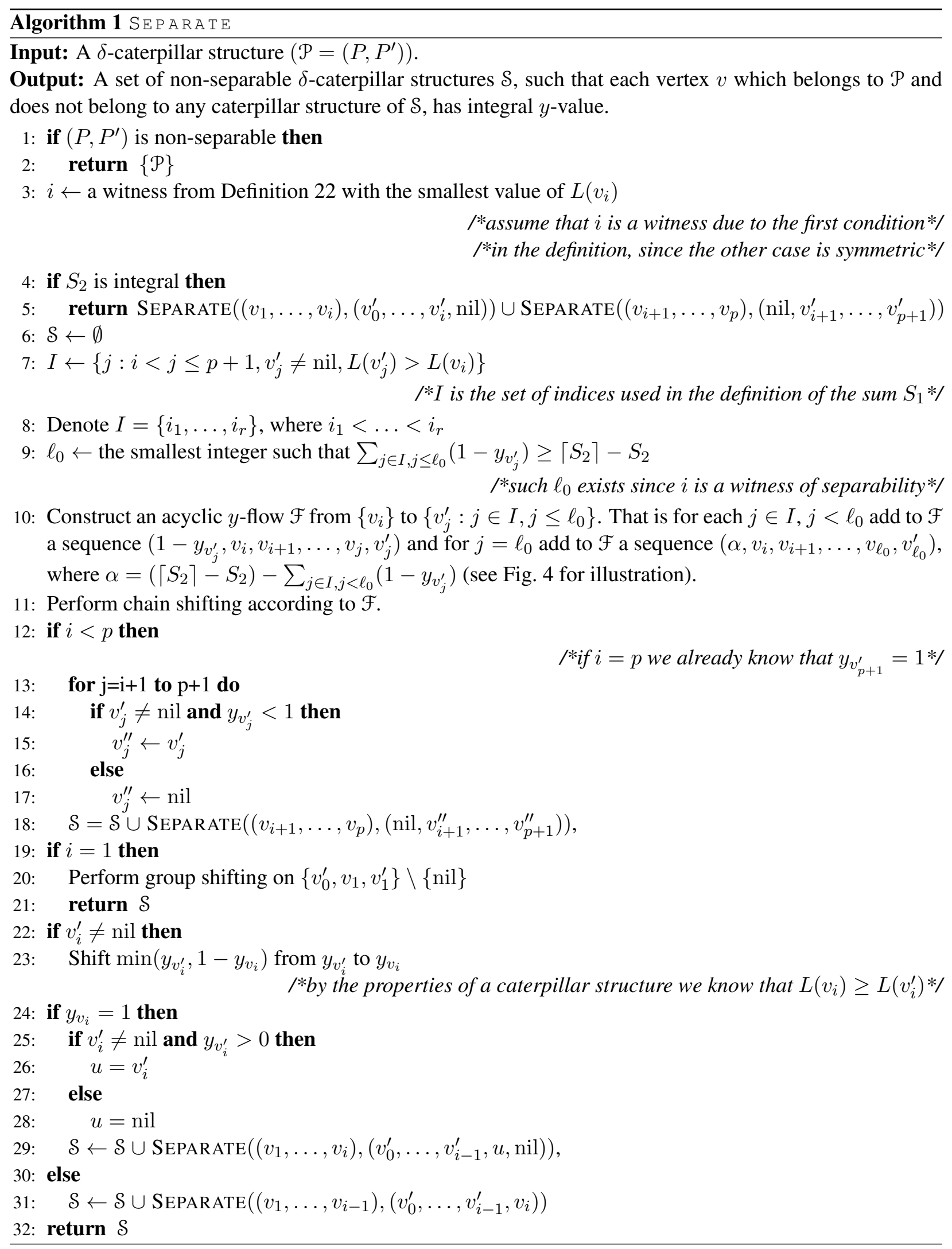


there is no $i<i^{\prime} \leq p+1$ with $v_{i}^{\prime} \neq$ nil and $L\left(v_{i}^{\prime}\right)>L\left(v_{i}\right)$. Note that for $\ell_{0}$ defined in Line 9 we have $\ell_{0} \leq j_{0}$ since due to the last property of good caterpillar structure $I \subseteq\left[i+1, j_{0}\right]$. Furthermore for each $j \in\left[i+1, \ell_{0}\right)$ either $v_{j}^{\prime}=$ nil or $L\left(v_{j}^{\prime}\right) \leq L\left(v_{i}\right)$ or after chain shifting by Lemma 20 the vertex $v_{j}^{\prime}$ will have integral $y$-value equal to one and will be not be included in caterpillar structures handled recursively. Consequently the caterpillar structure constructed in Line 18 is a good caterpillar structure. Finally consider caterpillar structures created in Lines 29 and 31 and observe that a pair of indices $i_{0}, i$ satisfies the properties of a good caterpillar structure.

Since all the caterpillar structures from the set $\mathcal{S}$ are good caterpillar structures the last part to show before we claim that $\left(x^{\prime}, y^{\prime}\right)$ is a 68 -feasible solution is that vertices from $X=\left(V\left(P_{0}\right) \cup V\left(P_{0}^{\prime}\right)\right) \backslash$ $\left(\bigcup_{\left(P, P^{\prime}\right) \in \mathcal{S}} V(P) \cup V\left(P^{\prime}\right)\right)$ have radius bounded by 68 (by the definition of a good caterpillar structure all vertices from $\bigcup_{\left(P, P^{\prime}\right) \in \mathcal{S}} V(P) \cup V\left(P^{\prime}\right)$ have radius bounded by 47$)$. The only possibilities for a vertex $v$ to become a member of $X$ is when its $y$-value becomes integral, which may happen in Line 17 (when $y_{v_{j}^{\prime}}^{\prime}=1$ ), Line 20 (for each non-nil vertex in $\left\{v_{0}^{\prime}, v_{1}, v_{1}^{\prime}\right\}$ ), Line 23 (when $y_{v_{i}^{\prime}}^{\prime}=0$ ). However in all cases because $i_{0}<i<j_{0}$ and by Lemma 20 we infer that radius $\left(x^{\prime}, y^{\prime}\right)(v) \leq 68$.

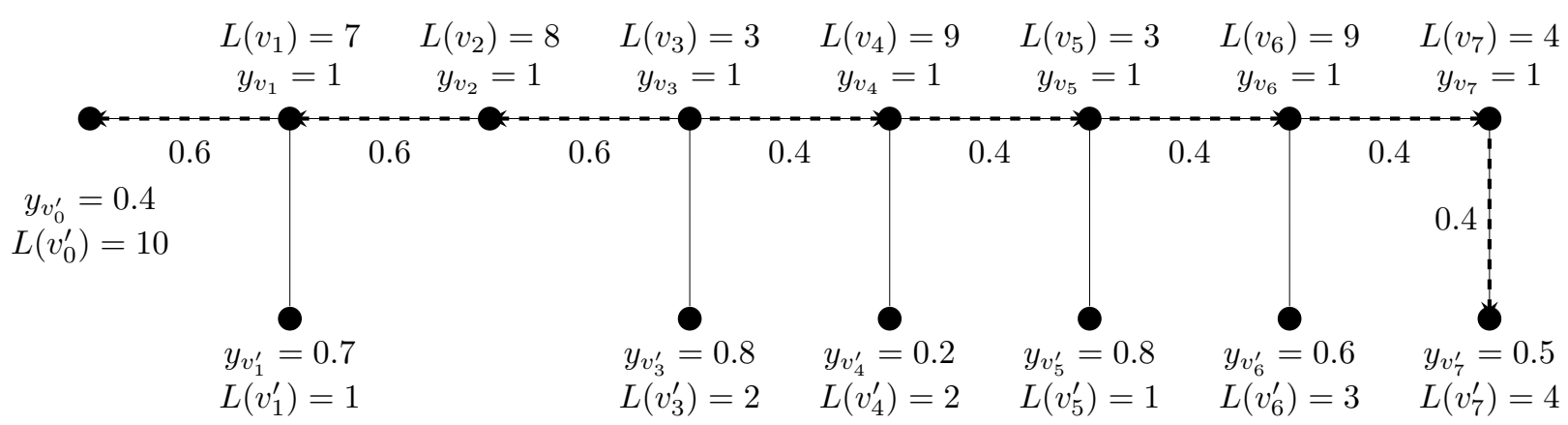

Figure 5: A dangerous caterpillar structure $\left(P=\left(v_{1}, \ldots, v_{7}\right), P^{\prime}=\left(v_{0}^{\prime}, v_{1}^{\prime}\right.\right.$, nil, $v_{2}^{\prime}, v_{3}^{\prime}, v_{4}^{\prime}, v_{5}^{\prime}, v_{6}^{\prime}, v_{7}^{\prime}$, nil $\left.)\right)$, where $\Gamma\left(\left(P, P^{\prime}\right)\right)=\left\{v_{3}, v_{5}\right\}$. The caterpillar structure is non-separable because both for $i=3$ and $i=5$ in Definition 22 the sum $S_{1}$ is at most 0.6, while $\left\lceil S_{2}\right\rceil-S_{2}$ is equal to 0.9. By dashed edges an acyclic flow $\mathcal{F}=\left\{\left(0.6, v_{3}, v_{2}, v_{1}, v_{0}^{\prime}\right),\left(0.4, v_{3}, v_{4}, v_{5}, v_{6}, v_{7}, v_{7}^{\prime}\right)\right\}$ from $\left\{v_{3}\right\}$ to $\left\{v_{0}^{\prime}, v_{7}^{\prime}\right\}$ is marked with values $f_{\mathcal{F}}$ printed in the middle of each arc.

In the following lemma we transform non-separable caterpillar structures into safe caterpillar structures.

Lemma 26. There exist constants $c, \delta$ such that for a given feasible LP solution $(x, y)$ we can find a $c$ feasible solution $\left(x^{\prime}, y^{\prime}\right)$ together with a set of vertex disjoint safe $\delta$-caterpillar structures $\mathcal{S}$ such that each vertex $v$ outside of the set has an integral $y$-value in $\left(x^{\prime}, y^{\prime}\right)$.

Proof. We use Lemma 25 to obtain a set $\mathcal{S}$ of vertex disjoint non-separable 21-caterpillar structures. Our goal is to transform each dangerous caterpillar structure in $\mathcal{S}$ into a safe caterpillar structure.

Consider a dangerous non-separable $\delta_{0}$-caterpillar structure $\mathcal{P}=\left(\left(v_{1}, \ldots, v_{p}\right),\left(v_{0}^{\prime}, \ldots, v_{p+1}^{\prime}\right)\right) \in \mathcal{S}$ and let $v_{a}$ be a minimum capacity vertex in $\Gamma(\mathcal{P})$. Moreover let $I=\left\{i: 0 \leq i \leq p+1 \wedge v_{i}^{\prime} \neq\right.$ nil $\wedge L\left(v_{i}^{\prime}\right)>$ $\left.L\left(v_{a}\right)\right\}$. Construct any acyclic $y$-flow which sends $\min \left(1, \sum_{i \in I}\left(1-y_{v_{i}^{\prime}}\right)\right.$ ) from $\left\{v_{a}\right\}$ to $\left\{v_{i}^{\prime}: i \in I\right\}$ (see Fig. 57. Such flow always exists due to Lemma 23 .

Let $Y=\left\{v_{a}, v_{a}^{\prime}, v_{a-1}^{\prime}\right\} \backslash\{$ nil $\}$ and perform group shifting on $Y$ (note that $a \geq 1$, since $v_{a} \in \Gamma(\mathcal{P})$ ). Replace $\mathcal{P}$ in $\mathcal{S}$ with the $\left(2 \delta_{0}\right)$-caterpillar structure $\left(\left(v_{1}, \ldots, v_{a-1}, v_{a+1}, \ldots, v_{p}\right)\right.$, $\left.\left(v_{0}^{\prime}, \ldots, v_{a-2}^{\prime}, u, v_{a+1}^{\prime}, \ldots, v_{p+1}^{\prime}\right)\right)$, where as $u$ we set the only vertex from $Y$ with fractional $y$-value after group shifting or we set $u=$ nil if all vertices in $Y$ have integral $y$-values. We need to argue, that when 
$u \neq$ nil, we have $L(u) \leq L\left(v_{a-1}\right)$, in order to satisfy (4) of Definition 16. Observe, that if $v_{a}^{\prime} \neq$ nil, then $L\left(v_{a}^{\prime}\right) \leq L\left(v_{a}\right)$, and similarly if $v_{a-1}^{\prime} \neq$ nil, then $L\left(v_{a-1}^{\prime}\right) \leq L\left(v_{a-1}\right)$. Hence to show $L(u) \leq L\left(v_{a-1}\right)$ it is enough to show $L\left(v_{a}\right) \leq L\left(v_{a-1}\right)$, but this follows from Lemma 23 , since $v_{a} \in \Gamma(\mathcal{P})$.

Note, that each caterpillar structure will be modified according to the above procedure at most twice, since after one iteration the sum $\sum_{i \in I}\left(1-y_{v_{i}^{\prime}}\right)$ either equals zero or decreases by one, and by Lemma 24 we have $\sum_{i \in I}\left(1-y_{v_{i}^{\prime}}\right)<2$. Consequently by Lemmas 20,14 we obtain the desired set of vertex disjoint $\delta$-caterpillar structure together with a $c$-feasible solution.

\subsection{Rounding safe caterpillar structures}

In this section we describe how to round the $c$-feasible solution $\left(x^{\prime}, y^{\prime}\right)$ using the set of vertex disjoint safe caterpillar structures $\mathcal{S}$ from Lemma 26. In order to do that we introduce a notion of rounding flow which is a special kind of $y$-flow defined for a caterpillar structure.

Definition 27 (rounding flow). For a caterpillar structure $\left(P, P^{\prime}\right)$ and an assignment $(x, y)$ we call $\mathcal{F}$ a rounding flow iff $\mathcal{F}$ is a $y$-flow from $S$ to $T$ where $S \cup T=V\left(P^{\prime}\right)$, for each $v_{i}^{\prime} \in S$ we have $f_{\mathcal{F}}\left(\left(v_{i}^{\prime}, v_{i}\right)\right)=y_{v_{i}^{\prime}}$ and for each $v_{i}^{\prime} \in T$ we have $f_{\mathcal{F}}\left(\left(v_{i}, v_{i}^{\prime}\right)\right)=1-y_{v_{i}^{\prime}}$. Furthermore each flow path from $\mathcal{F}$ can not go through a vertex from $V \backslash\left(V(P) \cup V\left(P^{\prime}\right)\right)$.

In order to obtain a rounding flow for each vertex of $V\left(P^{\prime}\right)$ (which by definition have fractional $y$ values), we have to decide whether it will be a source (member of $S$ ) or a sink (member of $T$ ). After chain shifting according to $\mathcal{F}$ all sources should have $y$-value equal to zero whereas all sinks should have $y$-value equal to one and consequently all vertices from the caterpillar structure will have integral $y$-value. In the following lemma we show that for each non-separable caterpillar structure we can always find a rounding flow in polynomial time.

Lemma 28. For any safe $\delta$-caterpillar structure $\left(P, P^{\prime}\right)$ and an assignment $(x, y)$ there exists a rounding flow $\mathcal{F}$ such that for any two adjacent vertices in $G_{\mathcal{F}}$ their distance in $G$ is at most $\delta$. Furthermore we can find such a rounding flow in polynomial time.

Proof. We present a recursive procedure which constructs a desired rounding flow. Note that some recursive calls of the procedure might potentially involve infeasible assignments $\left(x^{\prime}, y^{\prime}\right)$, however we prove that if the initial call gives the procedure a safe $\delta$-caterpillar structure, then as a result we obtain a valid rounding flow.

Let us describe a procedure which is given a caterpillar structure $\left(P, P^{\prime}\right)$ together with an assignment $y$ (the procedure does not need the $x$ part of an assignment). Denote $P=\left(v_{1}, \ldots, v_{p}\right)$ and $P^{\prime}=$ $\left(v_{0}^{\prime}, \ldots, v_{p+1}^{\prime}\right)$. If $V\left(P^{\prime}\right)=\emptyset$ then we simply return the empty rounding flow. Otherwise let $i$ be the smallest integer such that the sum of $y$-values of $X=\left\{v_{0}^{\prime}, \ldots, v_{i}^{\prime}\right\} \backslash$ nil is at least one (such $i$ always exists since the sum of all $y$-values in $V\left(P^{\prime}\right)$ is integral by $(8)$ of Def. 16. Note that since all vertices in $V\left(P^{\prime}\right)$ have fractional $y$-values we have $i>0$. Let $0 \leq i_{0} \leq i$ be an index such that $v_{i_{0}}^{\prime} \neq$ nil and $v_{i_{0}}^{\prime}$ has the biggest capacity in $X$. Let $\alpha=\sum_{v \in X} y_{v}$. If $\alpha=1$ then we recursively construct a rounding flow $\mathcal{F}$ from $S$ to $T$ for a smaller caterpillar structure $\left(\left(v_{i+1}, \ldots, v_{p}\right),\left(\right.\right.$ nil, $\left.\left.v_{i+1}^{\prime}, \ldots, v_{p+1}^{\prime}\right)\right)$ and (i) add to $S$ the set of vertices $X \backslash\left\{v_{i_{0}}^{\prime}\right\}$ (ii) add to $T$ the vertex $v_{i_{0}}^{\prime}$ (iii) for each $v_{j}^{\prime} \in X \backslash\left\{v_{i_{0}}^{\prime}\right\}$ add to $\mathcal{F}$ a flow path $\left(y_{v_{j}^{\prime}}, v_{j}^{\prime}, v_{j}, \ldots, v_{i_{0}}, v_{i_{0}}^{\prime}\right)$. In this case we return $\mathcal{F}$ as a desired rounding flow for $\left(P, P^{\prime}\right)$. Hence from now on we assume $\alpha>1$ and $\alpha-1<y_{v_{i}^{\prime}}$. Consider two cases: $i_{0}<i$ and $i_{0}=i$.

First let us assume that $i_{0}<i$. We store $z:=y_{v_{i}^{\prime}}$ and temporarily set $y_{v_{i}^{\prime}}=\alpha-1$. Next recursively construct a rounding flow $\mathcal{F}$ from $S \subseteq V\left(P^{\prime \prime}\right)$ to $T \subseteq V\left(P^{\prime \prime}\right)$ for a smaller caterpillar structure $\left(\left(v_{i}, \ldots, v_{p}\right), P^{\prime \prime}\right)$, where $P^{\prime \prime}=\left(\right.$ nil $\left., v_{i}^{\prime}, \ldots, v_{p+1}^{\prime}\right)$ (note that the sum of $y$-values in $P^{\prime \prime}$ is integral). Now consider two cases: 
- if $v_{i}^{\prime} \in S$ then: (i) add to $S$ vertices from $X \backslash\left\{v_{i}^{\prime}, v_{i_{0}}^{\prime}\right\}$ (ii) add to $T$ the vertex $v_{i_{0}}^{\prime}$ (iii) for each $v_{j}^{\prime} \in X \backslash\left\{v_{i_{0}}^{\prime}, v_{i}^{\prime}\right\}$ add to $\mathcal{F}$ a flow path $\left(y_{v_{j}^{\prime}}, v_{j}^{\prime}, v_{j}, \ldots, v_{i_{0}}, v_{i_{0}}^{\prime}\right)$ (iv) add to $\mathcal{F}$ a flow path $(z-$ $\left.y_{v_{i}^{\prime}}, v_{i}^{\prime}, v_{i}, \ldots, v_{i_{0}}, v_{i_{0}}^{\prime}\right)(\mathrm{v})$ set $y_{v_{i}^{\prime}}:=z(\mathrm{vi})$ return $\mathcal{F}$.

- if $v_{i}^{\prime} \in T$ then: (i) add to $S$ vertices from $X \backslash\left\{v_{i}^{\prime}, v_{i_{0}}^{\prime}\right\}$ (ii) add to $T$ the vertex $v_{i_{0}}^{\prime}$ (iii) out of the flow paths in $\mathcal{F}$ that end in $v_{i}^{\prime}$ leave only that many, that send exactly $1-z$ units of flow and reroute the rest paths to $v_{i_{0}}^{\prime}$ through vertices $v_{i-1}, v_{i-2}, \ldots, v_{i_{0}}$ (iv) for each $v_{j}^{\prime} \in X \backslash\left\{v_{i_{0}}^{\prime}, v_{i}^{\prime}\right\}$ add to $\mathcal{F}$ a flow path $\left(y_{v_{j}^{\prime}}, v_{j}^{\prime}, v_{j}, \ldots, v_{i_{0}}, v_{i_{0}}^{\prime}\right)(\mathrm{v})$ return $\mathcal{F}$.

Now assume that $i_{0}=i$. We create a smaller caterpillar structure $\left(\left(v_{a}, v_{i+1}, v_{i+2}, \ldots, v_{p}\right)\right.$, $\left(\right.$ nil, $\left.\left.v_{a}^{\prime}, v_{i+1}^{\prime}, \ldots, v_{p+1}^{\prime}\right)\right)$, where $v_{a}, v_{a}^{\prime}$ are two newly created vertices with $y_{v_{a}^{\prime}}:=\alpha-1$ and $L\left(v_{a}^{\prime}\right):=$ $L\left(v_{a}\right):=L\left(v_{i_{1}}^{\prime}\right)$, where $v_{i_{1}}^{\prime}$ is the second biggest capacity vertex in the set $X$. Next run recursively our procedure on the newly created caterpillar structure to obtain a rounding flow $\mathcal{F}$ from $S$ to $T$. Again, consider two cases:

- if $v_{a}^{\prime} \in S$ then: (i) set $S:=\left(S \backslash\left\{v_{a}^{\prime}\right\}\right) \cup\left(X \backslash\left\{v_{i}^{\prime}\right\}\right)$ (ii) set $T:=T \cup\left\{v_{i}^{\prime}\right\}$ (iii) change in $\mathcal{F}$ all the paths that start in $v_{a}^{\prime}$ to start in $X \backslash\left\{v_{i}^{\prime}\right\}$ (iv) add to $\mathcal{F}$ paths that start in $X$ and transfer $1-y_{v_{i}^{\prime}}$ units of flow from $X$ to $v_{i}^{\prime}(\mathrm{v})$ return $\mathcal{F}$.

- if $v_{a}^{\prime} \in T$ then: (i) set $S:=S \cup\left(X \backslash\left\{v_{i}^{\prime}, v_{i_{1}}^{\prime}\right\}\right)$ (ii) set $T:=\left(T \backslash\left\{v_{a}^{\prime}\right\}\right) \cup\left\{v_{i}^{\prime}, v_{i_{1}}^{\prime}\right\}$ (iii) reroute some of the flow paths from $\mathcal{F}$ that end in $v_{a}^{\prime}$ to that transfer exactly $1-y_{v_{i}^{\prime}}$ units of flow to $v_{i^{\prime}}$ (that is remove $v_{a}^{\prime}$ as the last vertex on those paths and extend the paths by $v_{i}, v_{i}^{\prime}$ ) (iv) reroute all the remaining flow paths in $\mathcal{F}$ that end in $v_{a}^{\prime}$ to $v_{i_{1}}^{\prime}$ (that is remove $v_{a}^{\prime}$ and extend those paths by $v_{i}, v_{i-1}, \ldots, v_{i_{1}}, v_{i_{1}}^{\prime}$ ) (v) for each $v_{j}^{\prime} \in X \backslash\left\{v_{i}^{\prime}, v_{i_{1}}^{\prime}\right\}$ add to $\mathcal{F}$ a flow path $\left(y_{v_{j}^{\prime}}, v_{j}^{\prime}, v_{j}, \ldots, v_{i_{1}}, v_{i_{1}}^{\prime}\right)(\mathrm{v})$ return $\mathcal{F}$.

Finally we prove that if the procedure receives a safe caterpillar structure then it returns a desired rounding flow. The only property of the rounding flow that needs detailed analysis is the assumption that each internal vertex of a flow path has capacity not smaller than its the capacity of its starting point. Let us assume that there exists a path in $\mathcal{F}$ that starts in $v_{a}^{\prime}$, goes though $v_{b}$ and ends in $v_{c}^{\prime}$, where $L\left(v_{c}^{\prime}\right) \geq L\left(v_{a}^{\prime}\right)>L\left(v_{b}\right)$. This contradicts the assumption that $\mathcal{P}$ is safe because $v_{b} \in \Gamma(\mathcal{P})$.

The following theorem summarizes Sections 2.1, 2.2, 2.3, 2.4.

Theorem 29. For a connected graph $G$, if LPI has a feasible solution then we can find a c-feasible solution with integral $y$-values.

Proof. Using a feasible solution to LP1, by Lemma 26, we obtain a $c$-feasible solution $\left(x^{\prime}, y^{\prime}\right)$, together with a set of vertex disjoint safe $\delta$-caterpillar structures $\mathcal{S}$, such that vertices that do not belong to any caterpillar structure in $\mathcal{S}$ have integral $y$-value in $\left(x^{\prime}, y^{\prime}\right)$. Next by Lemma 28 for each $\delta$-caterpillar structure $\left(P, P^{\prime}\right) \in \mathcal{S}$ we find a rounding flow $\mathcal{F}_{\left(P, P^{\prime}\right)}$. Finally for each $\delta$-caterpillar structure $\left(P, P^{\prime}\right)$ we perform chain shifting with respect to $\mathcal{F}_{\left(P, P^{\prime}\right)}$, and by Lemma 20 we obtain a $c^{\prime}$-feasible solution $\left(x^{\prime \prime}, y^{\prime \prime}\right)$ to LP1.

By Lemma 26, vertices outside of $\mathcal{S}$ have integral $y$-value in $\left(x^{\prime}, y^{\prime}\right)$. Moreover by Definition 27, after chain shifting all the vertices in each caterpillar structure of $\mathcal{S}$ have integral $y$-values in $\left(x^{\prime \prime}, y^{\prime \prime}\right)$.

\subsection{Rounding $x$-values}

In this section we show how to extend Theorem 29 to obtain not only integral $y$-values, but also integral $x$-values. The following lemma is standard (using network flows).

Lemma 30. Let $(x, y)$ be a $\delta$-feasible solution such that all $y$-values are integral. There is a polynomial time algorithm that creates a $\delta$-feasible solution which has both $x$-and $y$-values integral.

As a consequence of Theorem 29 and the above lemma the proof of Theorem 2 follows. 


\section{Soft capacities}

This section is devoted to the soft capacities variant, where one can open an arbitrary number of centers in a node. We present an 11-approximation algorithm for the soft capacitated version of the $k$-center problem with non-uniform capacities, i.e. we prove Theorem 4 Let us recall that for this problem the LP relaxation for the natural IP, which we denote as LP1 has the following form.

$$
\begin{aligned}
& \sum_{u \in V} y_{u}=k \\
& x_{u, v} \leq y_{u} \\
& \sum_{v \in V} x_{u, v} \leq L(u) y_{u} \\
& \sum_{u \in V} x_{u, v}=1 \\
& x_{u, v}=0 \\
& x_{u, v} \geq 0
\end{aligned}
$$

The following lemma is proved by Khuller and Sussman.

Lemma 31 ([22]). For a connected graph $G=(V, E)$ one can in polynomial time construct an inclusionwise maximal independent set $S \subseteq V$ in $G^{2}$, such that $G^{3}[S]$ is connected.

The proof of the main theorem of this section is inspired by the algorithm of [22] for uniform soft capacities.

Theorem 32. There is a polynomial time algorithm, which given an instance of the soft-capacitated $k$ center problem for a connected graph, and a fractional feasible solution for LP1, can round it to an integral solution that uses non-zero $x_{u, v}$ variables for pairs of nodes with distance at most 11 .

Proof. First, we construct the set $S$ using the algorithm from Lemma 31. Observe, that by constraints (15) and (13) for each $v \in V$ we have

$$
\sum_{u \in N[v]} y_{u} \geq \sum_{u \in N[v]} x_{u, v}=1
$$

Since $S$ is independent in $G^{2}$, by constraint 12 we infer that $|S| \leq k$.

We prove the theorem in two steps. First, we construct a function $\phi: V \rightarrow S$, such that for each $v \in V$ we have $\operatorname{dist}_{G}(v, \phi(v)) \leq 5$ and for each $s \in S$ we have $\left|\phi^{-1}(s)\right| \leq \max _{v \in V \text {, } \operatorname{dist}_{G}(v, s) \leq 6} L(v)$, that is we assign each vertex to an element of $S$ within distance 5 , but we increase the capacity of each $s$ to the maximum capacity reachable within distance 6 . In the second step we reassign vertices in such a way, that the maximum distance is at most 11 , and at the same time capacity constraints are satisfied.

Let $T$ be any spanning tree of $G^{3}[S]$, rooted at an arbitrary vertex $r \in S$. Consider the following procedure, which constructs a function $u: S \rightarrow \mathbb{R}_{+} \cup\{0\}$ assigning a tentative fractional number of centers to open in each of the vertices in $S$.

1. Initially set $u(s)=0$ for each $s \in S$.

2. For each vertex $v \in V$, if $\operatorname{dist}_{G}(v, S) \leq 1$, then by the fact that $S$ is independent in $G^{2}$ there exists exactly one vertex $s_{v} \in S$, such that $\operatorname{dist}_{G}\left(v, s_{v}\right) \leq 1$, otherwise (when $\operatorname{dist}_{G}(v, S)=2$ ), as $s_{v}$ set any vertex from $S$ within distance 2 from $v$.

3. For each vertex $v \in V$ increase $u\left(s_{v}\right)$ by $y_{v}$ (note that after this operation for each $s \in S$ we have $u(s) \geq 1$ and $\left.\sum_{s \in S} u(s)=k\right)$. 
4. For each vertex $s \in S$ in a bottom-up order with respect to $T$, let $x=u(s)-\lceil u(s)\rceil$, decrease $u(s)$ by $x$ and increase $u(p(s))$ by $x$, where $p(s)$ is the parent of $s$ in the tree $T$ (assume that $p(r)=r$ ).

The last step of the above process ensures, that the function $u$ has only integral positive values and moreover $\sum_{s \in S} u(s)=k$. We claim that if we open exactly $u(s)$ centers in a vertex $s$, for each $s \in S$, then there exists an assignment $\phi: V \rightarrow S$, such that for each $v \in V$ we have $\operatorname{dist}_{G}(v, \phi(v)) \leq 5$ and for each $s \in S$ we have $\left|\phi^{-1}(s)\right| \leq \max _{v \in V, \operatorname{dist}_{G}(v, s) \leq 6} L(v)$. Note that if we know that it exists, then we can find it in polynomial time by using maximum flow computation. To show the existence of $\phi$, observe that it is enough to show a function $f: V \times S \rightarrow \mathbb{R}_{+} \cup\{0\}$, satisfying:

- for each $v \in V$ we have $\sum_{s \in S} f(v, s)=y_{v}$,

- for each $s \in V$ we have $\sum_{v \in V} f(v, s)=u(s)$,

- for each $v \in V, s \in S$ if $f(v, s)>0$, then $\operatorname{dist}_{G}(v, s) \leq 5$.

Less formally, the function $f(v, *): S \rightarrow \mathbb{R}_{+} \cup\{0\}$ is a distribution of the value $y_{v}$ among vertices of $S$ within distance 5 . Such a function guarantees that we can fractionally cover all the vertices within distance 5 when we open an integral number of centers $u(s)$ in each vertex $s \in S$, therefore we can also cover vertices of $V$ integrally, which proves the existence of the desired assignment $\phi$. Observe, that we can construct the function $f$ while performing the bottom-up process in Step 4, where for each vertex $v \in V$ we split the value $y_{v}$ between $f\left(v, s_{v}\right)$ and $f\left(v, p\left(s_{v}\right)\right)$. It is always possible, because while going up the tree we can ensure that the part of $y_{v}$, that was assigned to $f\left(v, p\left(s_{v}\right)\right)$ remains in $p\left(s_{v}\right)$, since we send up $u(s)-\lceil u(s)\rceil<1$ and after Step 3 we have $u\left(s^{\prime}\right) \geq 1$ for each $s^{\prime} \in S$. Consequently, we know that there exists the desired assignment $\phi$.

Finally, we construct the final assignment $\phi_{0}: V \rightarrow S^{\prime}$ as follows. For each $s \in S$ let

$$
s_{L}=\arg \max _{v \in V, \operatorname{dist}_{G}(s, v) \leq 6} L(v),
$$

open $u(s)$ centers in $s_{L}$ and assign all the vertices of $\phi^{-1}(s)$ to $s_{L}$ in $\phi_{0}$. By the properties of $\phi$ we infer that in this way we obtain a multiset $S^{\prime}$ of exactly $k$ centers and an assignment $\phi_{0}$, satisfying $\operatorname{dist}_{G}\left(v, \phi_{0}(v)\right) \leq$ 11 for each $v \in V$ and $\left|\phi^{-1}\left(s^{\prime}\right)\right| \leq L\left(s^{\prime}\right)$ for each $s^{\prime} \in S$.

Corollary 33. The integrality gap of LP1 for connected graphs is at most 11 and there is a 11-approximation algorithm for connected graphs.

Since Theorem 5 shows that a $c$-approximation algorithm for connected graphs implies a $c$-approximation algorithm for general graphs, the proof of Theorem 4 follows.

\section{Uniform capacities}

Here, we prove Theorem 8 , i.e. we show 5 and 6 upper bounds for the integrality gap of LP1 for uniformsoft-capacities and uniform-hard-capacities respectively, which is a counterposition to lower bounds of 4 and 5 as stated in Theorem 7 .

The algorithm of Khuller and Sussmann [22] gives 6- and 5-approximation algorithm for the uniform capacitated $k$-center problem, for hard and soft capacities respectively. It is possible to reformulate this algorithm to make it a rounding algorithm for LP1. However to avoid rewriting the whole algorithm, we show that if the algorithm of [22] does not produce a solution (which means that there is no solution which uses at most one hop), then it produces a witness, showing that there is no solution using $k$ vertices and covering within distance of at most one hop. For a given instance $(G, k, L)$ of uniform capacitated $k$-center problem if the algorithm of Khuller and Sussmann does not produce a solution, both in the case of hard and soft capacities, then it creates a set $V_{0} \subseteq V(G)$, such that: 
(i) for any pair of vertices $u, v \in V_{0}$, the distance between $u$ and $v$ in $G$ is at least 3,

(ii) $\left|V_{0}\right|+\frac{\left|V^{\prime}\right|}{L}>k$, where $V^{\prime}=\left\{v \in V(G): \forall_{u \in V_{0}} \operatorname{dist}_{G}(u, v) \geq 3\right\}$.

Using the properties (i) and (ii) of $V_{0}$ we prove that there is no feasible solution for LP1. By (i) and by constraints (4) and (6) of LP1 we have:

$$
\left|V_{0}\right|=\sum_{v \in V_{0}} \sum_{u \in N_{G}[v]} x_{u, v} \leq \sum_{v \in V_{0}} \sum_{u \in N_{G}[v]} y_{u}=\sum_{v \in N_{G}\left[V_{0}\right]} y_{v},
$$

where the last equality follows from (i). We lower bound the sum of $y$-values of vertices of $N_{G}\left[V^{\prime}\right]=$ $V \backslash N_{G}\left[V_{0}\right]$ as follows.

$$
\frac{\left|V^{\prime}\right|}{L}=\sum_{v \in V^{\prime}} \sum_{u \in N_{G}[v]} \frac{x_{u, v}}{L} \leq \sum_{u \in N_{G}\left[V^{\prime}\right]} y_{u}
$$

The first equality follows from constraint (4) and (6) of LP1, whereas the inequality follows from (3) of LP1. Therefore by (18), (19) and (ii), we infer constraint (1) of LP1 is violated.

$$
\sum_{v \in V} y_{v}=\sum_{v \in N_{G}\left[V^{\prime}\right]} y_{v}+\sum_{v \in N_{G}\left[V_{0}\right]} y_{v} \geq\left|V_{0}\right|+\frac{\left|V^{\prime}\right|}{L}>k
$$

Hence there is no feasible solution for LP1, which finishes the proof of Theorem 8 .

\section{Integrality gap lower bounds}

In this section we present lower bounds on the integrality gap of LP1, i.e. prove Theorem 7 .

We start with a construction for uniform hard capacities. Let $k \geq 24$ be an integer and set the uniform capacity as $L=k-1$. Let $G^{\prime}$ be a graph which consists of two adjacent vertices $a, b$ together with $L+2$ vertices adjacent to both $a$ and $b$. Let $G$ be a graph composed of:

- $k-6$ copies of the graph $G^{\prime}$ denoted as $G_{i}$ for $i=1, \ldots, k-6$,

- a single star $S_{k-6}$, rooted at $r$, which has exactly $k-6$ leaves denoted as $\ell_{1}, \ldots, \ell_{k-6}$,

- $k-6$ vertices $x_{i}$ for $i=1, \ldots, k-6$,

- $k-6$ edges $x_{i}, \ell_{i}$,

- for each $i=1, \ldots, k-6$ two edges between $a_{i}, b_{i} \in V\left(G_{i}\right)$ and $x_{i}$.

Note that the graph $G$ is connected. Observe that by setting the $y$-value equal to 1 for the vertex $r$, and $y_{a_{i}}=y_{b_{i}}=\frac{L+5}{2 L} \leq 1$ for each $i=1, \ldots, k-6$, we can set $x$ variables appropriately to obtain a feasible solution to the LP relaxation since the sum of $y$ variables is equal to $1+2(k-6) \frac{L+5}{2 L}=1+\frac{(k+4)(k-6)}{k-1} \leq$ $1+\frac{(k-1)^{2}}{k-1} \leq k$.

Let us assume that there is a hard-capacitated $k$-center in the graph $G^{4}$. By $B_{i}$ (for $1 \leq i \leq k-6$ ) let us denote the set of vertices of $G_{i}$ of degree two. Consider any $1 \leq i \leq k-6$, and let us focus on how vertices of $B_{i}$ can be covered. Observe that the only vertices in $G$ that are within distance 4 from $B_{i}$ are $V\left(G_{i}\right) \cup\left\{x_{i}, \ell_{i}, r\right\}$. Since $\left|B_{i}\right|=L+2>L$, at least two centers are opened in the set $V\left(G_{i}\right) \cup\left\{x_{i}, \ell_{i}, r\right\}$, which mean that at least one center is opened in $V\left(G_{0}\right) \cup\left\{x_{i}, \ell_{i}, r\right\}$. Furthermore for different values of $i$, the sets $V\left(G_{i}\right) \cup\left\{x_{i}, \ell_{i}\right\}$ are disjoint. Moreover if only one center is opened in 


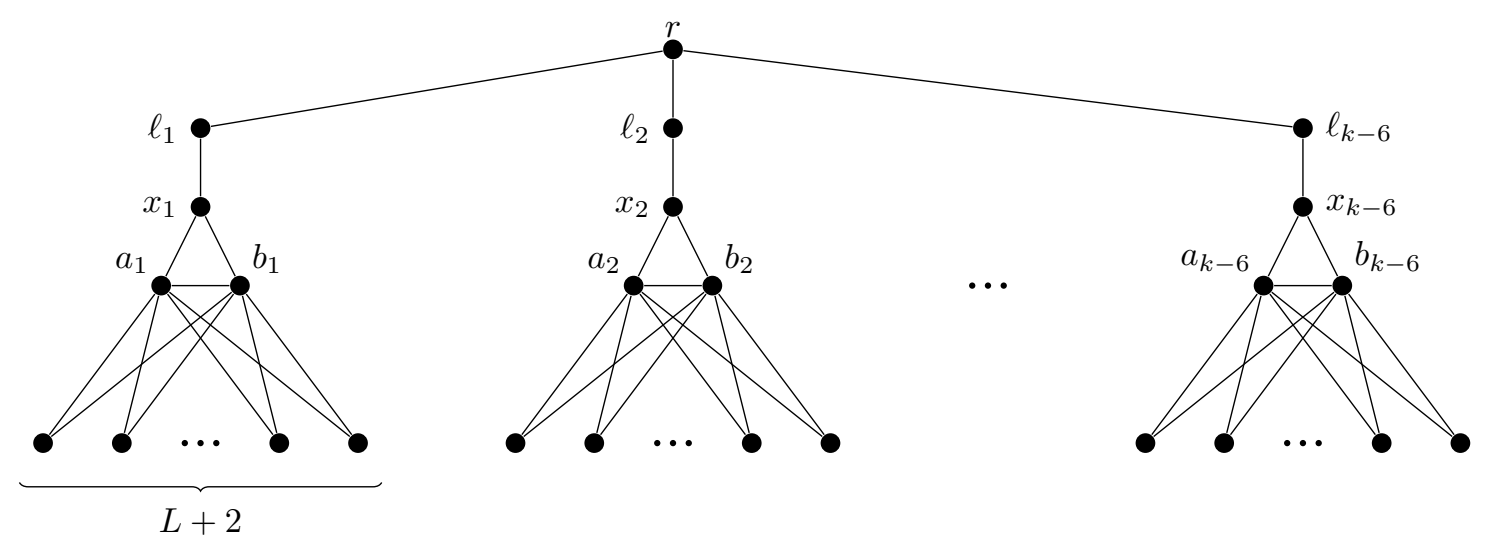

Figure 6: The graph $G$ used in the proof of Theorem 7 .

the set $V\left(G_{i}\right) \cup\left\{x_{i}, \ell_{i}\right\}$, then at least two vertices of $B_{i}$ have to be covered by $r$. Hence even if a center is opened in $r$, then for at least $(k-6)-\frac{L}{2}$ values of $i$ we need to open at least two centers in the set $V\left(G_{i}\right) \cup\left\{x_{i}, \ell_{i}\right\}$. Moreover we need to open at least one center in each of the sets $V\left(G_{i}\right) \cup\left\{x_{i}, \ell_{i}\right\}$, since otherwise $L+2$ vertices can not be covered. In total there are at least $(k-6)+\left((k-6)-\frac{L}{2}\right)$ centers opened, but $(k-6)+\left((k-6)-\frac{L}{2}\right)=k+(k-12)-\frac{k-1}{2}=k+\frac{k-23}{2}>k$ since $k \geq 24$, a contradiction.

For the uniform-soft-capacitated case the analysis is even simpler since if $G^{3}$ admits a soft-capacitated $k$-center then without loss of generality there are at least two centers open in each of the sets $V\left(G_{i}\right) \cup\left\{x_{i}, \ell_{i}\right\}$.

To prove the lower bound for non-uniform capacities, in the above example we change capacities to zero for all the vertices except $r$ and each of the $2(k-6)$ vertices $a_{i}, b_{i}$. Note that those are the only vertices with non-zero $y$-value in the constructed feasible solution for LP1. Moreover we have shown that in any integral solution there exists a vertex of $B_{i}$, which has to be covered by a vertex of $V\left(G_{j}\right) \cup\left\{x_{j}, \ell_{j}\right\}$, for $j \neq i$, and since the only vertices of non-zero capacity in this set are the vertices $a_{j}, b_{j}$, we infer, that some vertex of $B_{i}$ has to be covered by a vertex at distance at least 7 . Consequently the proof of Theorem 7 follows.

\section{$6 \quad(3-\epsilon)$-approximation hardness}

In this section we show, that it is not possible to approximate the $k$-center problem with non-uniform capacities in polynomial time with approximation ratio $(3-\epsilon)$ for constant $\epsilon>0$.

Theorem 34. If there exists a $(3-\epsilon)$-approximation algorithm for the $k$-center problem with non-uniform capacities, where all the non-zero capacities, then $P=N P$. The theorem applies both to hard and soft capacitated version.

Proof. Let us assume that a $(3-\epsilon)$-approximation algorithm exists. We present a reduction from the EXACT COVER BY 3-SETS problem, which is NP-complete.

\section{EXACT COVER BY 3-SETS}

Input: A set system $(\mathcal{F}, U)$, where each set in $\mathcal{F}$ has exactly 3 elements.

Question: Does there exist a subset $\mathcal{F}^{\prime} \subseteq \mathcal{F}$, such that each element of the universe $U$ belongs to exactly one set in $\mathcal{F}^{\prime}$.

Let $I=(\mathcal{F}, U)$ be an instance of EXACT COVER BY 3-SETS. As a graph $G$ we take the bipartite graph $(\mathcal{F} \cup B, E)$, where $B=\bigcup_{i=1}^{|\mathcal{F}|+1} U_{i}, U_{i}=\left\{u_{i}: u \in U\right\}$ and $E=\left\{S u_{i}: S \in \mathcal{F} \wedge 1 \leq i \leq|\mathcal{F}|+1 \wedge u \in S\right\}$. 
That is $G$ is an incidence graph of the set system $(\mathcal{F}, U)$, where the universe is replicated $|\mathcal{F}|+1$ times. Additionally for each $S \in \mathcal{F}$ we add to the graph a vertex $x_{S}$, which is adjacent to $S$, and has exactly $3|\mathcal{F}|+1$ pendant neighbors (see Fig. 7). We set a capacity function $L: V(G) \rightarrow \mathbb{Z}_{+} \cup\{0\}$ as follows:

- For each $u_{i} \in U_{i}, L\left(u_{i}\right)=0$.

- For each $S \in \mathcal{F}, L(S)=L\left(x_{S}\right)=3|\mathcal{F}|+3$.

- For each pendant vertex $v$, which is adjacent to $x_{S}$, for $S \in \mathcal{F}$, we set $L(v)=0$.

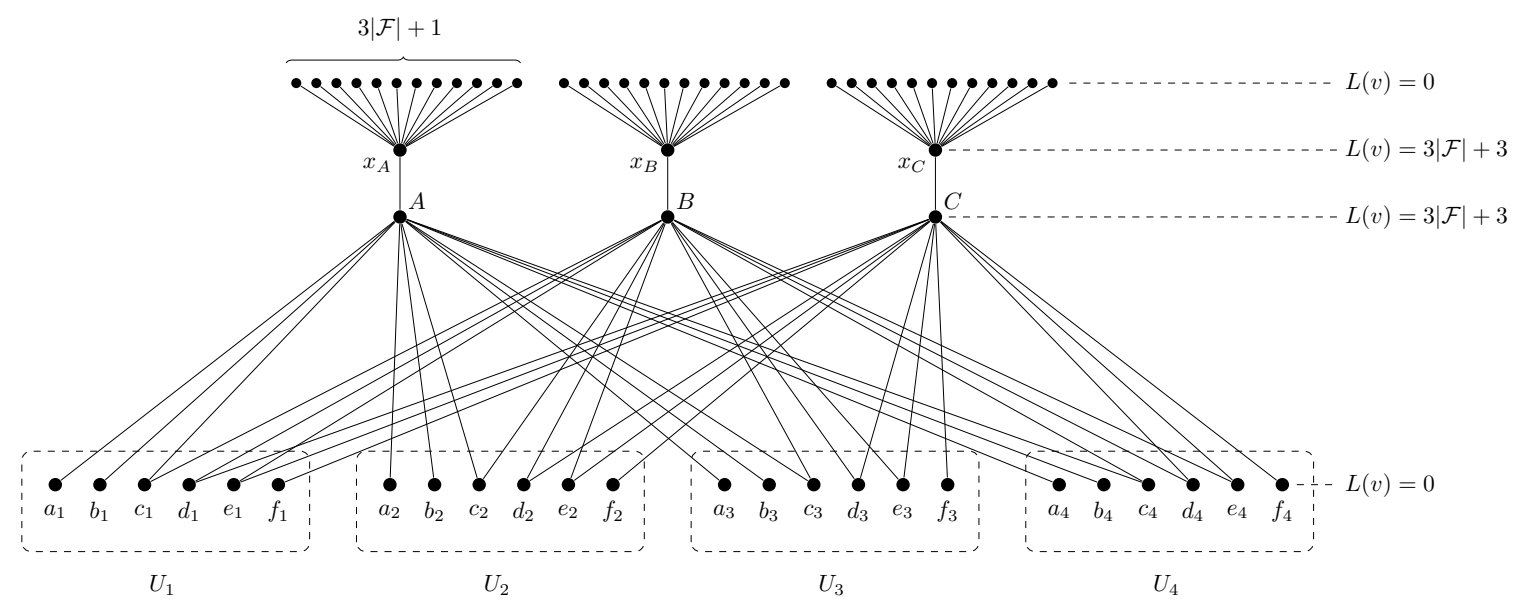

Figure 7: The graph $G$ constructed for the set system $(\mathcal{F}, U)$, where $\mathcal{F}=\{\{A=\{a, b, c\}, B=$ $\{c, d, e\}, C=\{d, e, f\}\}\}$ and $U=\{a, b, c, d, e, f\})$

Note that all the vertices, which have non-zero capacities, have exactly the same capacity. Let $I^{\prime}=$ $(G, L, k=|\mathcal{F}|+|U| / 3)$ be an instance of the capacitated $k$-center problem. In what follows we prove:

(i) If $I$ is a YES-instance, then there exists a set $V_{0} \subseteq V(G)$, such that $\left|V_{0}\right|=k$, together with a function $\phi: V \rightarrow V_{0}$, which satisfies $\forall_{v \in V(G)} \operatorname{dist}_{G}(v, \phi(v)) \leq 1$ and $\forall_{v \in V_{0}}\left|\phi^{-1}(v)\right| \leq L(v)$. Less formally, $V_{0}$ is a solution for the capacitated $k$-center problem, which opens at most one center in each vertex.

(ii) Let $V_{0}$ be a multiset containing exactly $k$ vertices of $V(G)$, such that there exists a function $\phi$ : $V \rightarrow V_{0}$, which satisfies $\forall_{v \in V(G)} \operatorname{dist}_{G}(v, \phi(v))<3$ and $\forall_{v \in V_{0}}\left|\phi^{-1}(v)\right| \leq L(v)$. Then one can in polynomial time construct a set $\mathcal{F}^{\prime} \subseteq \mathcal{F}$, such that each element of the universe $U$ belongs to exactly one set in $\mathcal{F}^{\prime}$. Intuitively, given a solution to the capacitated $k$-center problem which uses distances at most two, and potentially opens more than one center in a vertex, one can in polynomial time construct a solution for the instance $I$.

Observe that having (i) and (ii) suffices to prove the theorem, since if $I$ is a YES-instance, we can obtain a solution to $I$, by constructing the graph $G$, running the $(3-\epsilon)$-approximation algorithm on $(G, L, k)$, which by (i) returns a set $V_{0}$, which by (ii) we transform to a solution for $I$ in polynomial time.

First we prove (i). Let $\mathcal{F}^{\prime}$ be a solution for the instance $I$. We take $V_{0}=\mathcal{F}^{\prime} \cup\left\{x_{S}: S \in \mathcal{F}\right\}$, that is $|U| / 3$ sets from $\mathcal{F}$, and all the vertices $x_{S}$. Note that $\left|V_{0}\right|=|U| / 3+|\mathcal{F}|$ and each vertex in $V_{0}$ has capacity exactly $3|\mathcal{F}|+3$. Observe that if each vertex $S \in \mathcal{F}^{\prime}$ covers each of the $|\mathcal{F}|+1$ copies of the three elements in $S$, while each vertex $x_{S}$ covers itself, all the $3|\mathcal{F}|+1$ pendant vertices, and the vertex $S$, then we obtain the desired function $\phi$. 
Next we prove (ii). Observe, that $|V(G)|=(|\mathcal{F}|+1)|U|+|\mathcal{F}|(3|\mathcal{F}|+3)=(3|\mathcal{F}|+3)(|U| / 3+|\mathcal{F}|)$, and hence in the multiset $V_{0}$ there are only vertices of capacity $3|\mathcal{F}|+3$, since otherwise the total capacity of the vertices in $V_{0}$ would be smaller than $|V(G)|$. Moreover, without loss of generality we can assume that the multiset $V_{0}$ contains only vertices $S \in \mathcal{F}$, since we can always replace a vertex $x_{S}$ by $S$ without exceeding distance two, in the function $\phi$. Furthermore pendant vertices of any $x_{S}$ are covered by vertices at distance at most two, that is by the vertex $S$. Therefore each $S \in \mathcal{F}$ appears in the multiset $V_{0}$ at least once. Let $\mathcal{F}^{\prime} \subseteq \mathcal{F}$ contain exactly those sets $S \in \mathcal{F}$, for which the vertex $S$ belongs to $V_{0}$ more than once. Note that since $\left|V_{0}\right|=|\mathcal{F}|+|U| / 3$, the set $\mathcal{F}^{\prime}$ contains at most $|U| / 3$ sets. Consequently to prove (ii) it is enough to show that each element of the universe $U$ belongs to at least one set in $\mathcal{F}^{\prime}$.

Let $V_{0}^{\prime} \subseteq V_{0}$ be the set containing the first copy of each $S \in \mathcal{F}$ in $V_{0}$, namely $V_{0}^{\prime}=\mathcal{F}$. Without loss of generality, for each $S \in V_{0}^{\prime}$ the set $\phi^{-1}(S)$ contains $x_{S}$ and all its pendant neighbors, since none of those vertices can be covered by $S^{\prime} \neq S$, because the distance would be at least three. Consequently for each $S \in V_{0}^{\prime}$ we have $\left|\phi^{-1}(S) \cap\left(\bigcup_{i=1}^{|\mathcal{F}|+1} U_{i}\right)\right| \leq L(S)-(3|\mathcal{F}|+2)=1$. Since $\left|V_{0}^{\prime}\right| \leq|\mathcal{F}|<|\mathcal{F}|+1$, there exists an index $1 \leq i_{0} \leq|\mathcal{F}|+1$, such that for each $S \in V_{0}^{\prime}$ we have $\phi^{-1}(S) \cap U_{i}=\emptyset$. Therefore each vertex $u_{i} \in U_{i}$ is covered by some set $S \in \mathcal{F}^{\prime}$ that it belongs to, which proves (ii) and finishes the proof of Theorem 34 .

\section{Conclusions and open problems}

We have obtained the first constant approximation ratio for the $k$-center problem with non-uniform hard capacities. The approximation ratio we obtain is in the order of hundreds (however we do not calculate it explicitly), so the natural open problem is to give an algorithm with a reasonable approximation ratio. Moreover, we have shown that the integrality gap of the standard LP formulation for connected graphs in the uniform capacities case is either 5 or 6 , which we think might be an evidence, that it should be possible to narrow the gap between the known lower bound of $(2-$ eps $)$ and upper bound 6 in the uniform capacities case.

\section{Acknowledgements}

We are thankful to anonymous referees for their helpful comments and remarks.

\section{References}

[1] V. Arya, N. Garg, R. Khandekar, K. Munagala, and V. Pandit. Local search heuristics for $k$-median and facility location problems. In Proc. of STOC'01, pages 21-29, 2001.

[2] J. Bar-Ilan, G. Kortsarz, and D. Peleg. How to allocate network centers. Journal of Algorithms, 15:385-415, 1993.

[3] Y. Bartal, M. Charikar, and D. Raz. Approximating min-sum k-clustering in metric spaces. In Proc. of STOC'01, pages 11-20, New York, NY, USA, 2001. ACM.

[4] M. H. Bateni and M. T. Hajiaghayi. Assignment problem in content distribution networks: unsplittable hard-capacitated facility location. ACM Trans. Algorithms. To appear. A preliminary version appeared in Proc. of SODA'09, 2009, pages 805-814. 
[5] J. Byrka. An optimal bifactor approximation algorithm for the metric uncapacitated facility location problem. In Proc. of APPROX'07.

[6] M. Charikar, S. Guha, É. Tardos, and D. Shmoys. A constant-factor approximation algorithm for the $k$-median problem. In Proc. of STOC'99.

[7] F. A. Chudak and D. P. Williamson. Improved approximation algorithms for capacitated facility location problems. Math. Program., 102(2, Ser. A):207-222, 2005.

[8] J. Chuzhoy, S. Guha, E. Halperin, S. Khanna, G. Kortsarz, R. Krauthgamer, and J. Naor. Asymmetric -center is $\log ^{*}$-hard to approximate. J. ACM, 52(4):538-551, 2005.

[9] J. Chuzhoy and J. Naor. Covering problems with hard capacities. In Proc of. FOCS'02, pages 481-489, 2002.

[10] J. Chuzhoy and Y. Rabani. Approximating k-median with non-uniform capacities. In Proc. of SODA'05, pages 952-958, 2005.

[11] R. Gandhi, E. Halperin, S. Khuller, G. Kortsarz, and A. Srinivasan. An improved approximation algorithm for vertex cover with hard capacities. JCSS, 72(1):16-33, 2006.

[12] R. Gandhi, S. Khuller, S. Parthasarathy, and A. Srinivasan. Dependent rounding and its applications to approximation algorithms. J. ACM, 53(3):324-360, 2006.

[13] M. R. Garey and D. S. Johnson. Computers and intractability. Freeman, 1979.

[14] T. Gonzalez. Clustering to minimize the maximum inter-cluster distance. Theoretical Computer Science, 38:293-306, 1985.

[15] S. Guha, R. Hassin, S. Khuller, and E. Or. Capacitated vertex covering with applications. J. Algorithms, 48(1):257-270, 2003.

[16] S. Guha and S. Khuller. Greedy strikes back: Improved facility location algorithms. Journal of Algorithms, 31:228-248, 1999.

[17] D. Hochbaum and D. Shmoys. A best possible heuristic for the $k$-center problem. Mathematics of Operations Research, 10:180-184, 1985.

[18] D. Hochbaum and D. Shmoys. A unified approach to approximation algorithms for bottleneck problems. Journal of the ACM, 33:533-550, 1986.

[19] W. Hsu and G. Nemhauser. Easy and hard bottleneck location problems. Discrete Applied Mathematics, 1:209-216, 1979.

[20] K. Jain and V. V. Vazirani. Approximation algorithms for metric facility location and $k$-median problems using the primal-dual schema and Lagrangian relaxation. Journal of the ACM, 48(2):274-296, 2001.

[21] J. J. Karaganis. On the cube of a graph. Canad. Math. Bull., 11:295-296, 1968.

[22] S. Khuller and Y. J. Sussmann. The capacitated k-center problem. SIAM J. Discrete Math., 13(3):403418, 2000. 
[23] M. R. Korupolu, C. G. Plaxton, and R. Rajaraman. Analysis of a local search heuristic for facility location problems. Journal of Algorithms, 37(1):146-188, 2000.

[24] R. Levi, D. B. Shmoys, and C. Swamy. LP-based approximation algorithms for capacitated facility location. In Proc. of IPCO'04, pages 206-218, 2004.

[25] S. Li. A 1.488 approximation algorithm for the uncapacitated facility location problem. In Proc. of ICALP'11, pages 77-88, 2011.

[26] J. Lin and J. Vitter. $\epsilon$-approximations with minimum packing constraint violation. In Proc. of STOC'92, pages $771-782,1992$.

[27] M. Mahdian and M. Pál. Universal facility location. In Proc. of ESA'03, pages 409-421, 2003.

[28] M. Pál, É. Tardos, and T. Wexler. Facility location with nonuniform hard capacities. In Proc. of FOCS'01, pages 329-338, 2001.

[29] M. Sekanina. On an ordering of the set of vertices of a connected graph. Technical Report Publ. Fac. Sci. Univ. Brno, 412, 1960.

[30] D. Shmoys, E. Tardos, and K. Aardal. Approximation algorithms for facility location problems. In Proc. of STOC'97, pages 265-274, 1997.

[31] J. Zhang, B. Chen, and Y. Ye. A multi-exchange local search algorithm for the capacitated facility location problem. In Proc. of IPCO'04, pages 219-233, 2004. 